\title{
Novel electrostatic devices for managing biotic and abiotic nuisances in environments
}

\begin{abstract}
Background: Principles of basic electrostatics have been used to develop new novel apparatuses for managing biotic and abiotic invasive nuisances in different environments. Biotic targets were air-borne spores of plant pathogenic fungi and pollen grains causing pollinosis and insect pests transmitting pathogens of crop plants, livestock and humans, and as an abiotic target smoke fine particle causing various human diseases. To control these environmental nuisances, unique phenomena generated in the electric field were applied to the apparatuses to capture or repel the targets. The discharge-free electric field, which was generated between the opposite charges of the earthed non-insulated conductor and the insulated conductor charged, was highly effective to create an electrostatic attractive force to trap biotic targets. The discharge-generating electric field was an alternative method to create the attractive force by negatively ionizing smoke fine particles in the electric field, to kill pathogenic microbials by exposing streamer discharge-based ozone or corona discharge-based plasma stream to them, and to electrocute insect pests by shooting high-energy free electrons to them. These theoretical and technical progresses enable not only to create spore-free and pest-free space in agricultural and horticultural environments for crop production and preservation, but to purify indoor air in human domestic environments.
\end{abstract}

Keywords: electric field screen, attractive force, insect pests, fungal spores, pollens, smoke particulate matter
Volume 2 Issue 5 - 2018

\author{
Yoshinori Matsuda,' Hideyoshi Toyoda ${ }^{2}$ \\ 'Laboratory of Phytoprotection Science and Technology, Faculty \\ of Agriculture, Kindai University, Nara 63I-8505, Japan \\ ${ }^{2}$ Research Association of Electric Field Screen Supporters, Nara \\ $63 \mathrm{I}-8505$, Japan
}

Correspondence: Hieyoshi Toyoda, Research Association of Electric Field Screen Supporters, Nara, 63I-8505, Japan, Tel +8I742-43-5223, Email: toyoda@enara.kindai.ac.jp

Received:September 22, 2018 | Published: September 26, 2018

\section{Introduction}

The protection of crop plants from infection and/or attack by pathogens and pests via safe and environmentally benign methods has been a long-standing goal. Much effort has focused on developing biological and chemical methods to achieve this, including the production of resistant crop plants using conventional and new biotechnological techniques, biocontrol of pathogens and pests using antibacterial, antifungal and entomopathogenic microorganisms, and the screening of biologically synthesised compounds that inhibit growth of the pathogens. ${ }^{1,2}$ Despite much interesting work, there has been little practical progress because the protective effects are easily destroyed, and because of problems with agent preparation, limited targets for application, and high costs. The principal problems facing practical implementation are the application of individual methods for pathogen and pest control at scales larger than the test experiments, and variable environmental conditions. These trials have taught us that the aforementioned techniques were, in essence, supplementary measures for a limited range of targets under particular conditions. We must reflect on the lack of reliable basic methods that can be combined to form an appropriate approach. ${ }^{1}$ Once a realistic and reasonable research objective can be settled, it would be possible to advance our steps steadily through a cyclical repetition of creation and destruction of a working hypothesis formulated from the reproducible experimental results and eventually to propose new conceptions for an electrostatic approach. A number of physical methods, especially exploiting electrostatic phenomena, can generate physical forces sufficiently strong to catch airborne fungal spores or small flying insects that may pass through the conventional insect netting used to protect greenhouse crop plants. If an effective force could be generated, such an electrostatic approach would be an extremely promising tool to provide a spore-free and pest-free space for crop plants in a greenhouse environment. ${ }^{3}$

In this review article, we describe the development of several types of electric field screen, including unique structures and electrostatic mechanisms for capturing insects and pathogens, focusing on the formation of a non-uniform static electric field. Each publication that we have made for this purpose provides diagrams and photographs, and in addition, videos are included to aid the reader in understanding how interesting the electrostatic world can be. Furthermore, we describe discharge-mediated electrostatic disinfection devices and spore collection apparatus based on dielectrophoretic particulate attraction. Moreover, an insatiable curiosity of electrostatic phenomena enabled to bring birth to new electrostatic apparatuses that can control other aerial biotic and abiotic targets such as pollen grains causing pollinosis and smoke fine particles involved in tobacco sidestream in human life environment. All related publications in the reference list of this article are self-contained, and these references can be read in any order, but these unfamiliar with screen structures are recommended to read chapters from the first to end according to the order of this article. We hope that this publication will provide an effective guide for the reader to the unique and fascinating world of electrostatics.

\section{Environmental nuisances controlled by the electrostatic approaches}

\section{Fungal spores}

The first environmental nuisance is airborne conidia (asexual spores) of plat pathogenic fungi, especially the prolific fungal pathogens causing diseases in pre- and post-harvest agricultural crops. 
Based on this criterion, airborne conidia of powdery mildew and blue mold are adopted as the model fungal pathogens that scatter about infectious spores in the air. Powdery mildew is a fungal pathogen that affects a wide range of plants. Powdery mildew diseases are caused by many different species of fungi in the order Erysiphales, which form large numbers of asexual progeny spores. In general, powdery mildew grows well in environments with high humidity and moderate temperatures. Greenhouses provide an ideal moist, temperate environment for the spread of the disease. Our major concern is the powdery mildew pathogen (Oidium neolycopersici) of greenhouse tomatoes; the pathogen severely infects not only all commercial tomato cultivars tested, ${ }^{4}$ but also the cultivars bred for resistance against a European isolate of the tomato powdery mildew pathogen. ${ }^{5}$ In our preliminary survey, we found fungicide-tolerant isolates of $O$. neolycopersici on naturally infected tomato leaves, indicating the necessity for alternative measures to control the pathogen. Although breeding resistant traits against the pathogen has been the conventional method to protect crop plants from the disease, ${ }^{6-8}$ we should always be alert for the outbreak of new pathogenic strains of the pathogen on newly bred resistant tomatoes. ${ }^{9-11}$ We thus have developed new physical electrostatics-based control measures to prevent the spread of the disease. ${ }^{12-15}$

Blue mold (by several species of genus Penicillium) is a common postharvest disease on fruit crops worldwide. This disease is an economic concern not only to the fresh-fruit industry but also to the fruit-processing industry. In addition, the blue mold is often found growing on household materials like wallpaper, insulation, and carpeting that have been damaged by water, and more importantly on valuable books in library. In fact, one of the most important precautions to safely preserve books in a library stack room is to keep the books from getting moldy. Mold develops easily in poorly ventilated rooms, and thus improving the ventilation in the stack room is a basic measure for avoiding mold. However, large arrays of filled bookshelves are likely to be an obstacle to even airflow in the room. Molding is most serious in older books because mold-proofing paper materials that inhibit colonization by mold were not used. Anti-mold treatments have been used widely in recent books, ${ }^{16}$ and more than $55 \%$ of our library-stocked books are mold-resistant. Our historically valuable books, however, are considerably old and are highly sensitive to mold infection. In fact, a thin film of mold has frequently grown over the entire surface of the books. To protect these books, we have been forced to conduct the costly and time-consuming fungicidal operations of periodically disinfecting the stack room with fumigants and routinely cleaning individual books by directly removing surface colonies with an alcohol-impregnated cotton wool. To solve this problem, it became essential to develop an alternative method to exclude airborne fungal spores from the space in which books were being preserved. For this purpose, we constructed a bookshelf surrounded by an electrostatic device that generates an air-permeable shield that prevents spores from entering the bookshelf. ${ }^{17}$ For a spore exclusion assay, we targeted cellulolytic fungi of genus Penicillium that are common causes of paper spoilage ${ }^{18} P$. digitatum was selected from our laboratory fungal stocks as a model of causal airborne fungi. These fungi are suitable for the preparation of abundant spores (conidia) because of their vigorous spore production. ${ }^{19}$ The electric field screen is a promising tool for physically excluding wind-carried fungal spores from a bookshelf. ${ }^{17}$

\section{Insect pests}

The second group of biotic environmental nuisances are a wide range of ubiquitous insect pests classified into different taxonomic groups. Whitefly (Bemisia tabaci), green peach aphid (Myzus persicae), western flower thrips (Frankliniella occidentalis), tomato leaf-miner fly (Liriomyza sativae), greenhouse shore fly (Scatella stagnalis) and green rice leaf hopper (Nephotettix cincticeps), all of which are pests of greenhouses and/or field crops. ${ }^{20,21}$ Vinegar fly (Drosophila melanogaster), red flour beetle (Tribolium castaneum), rice weevil (Sitophilus oryzae), Adzuki bean weevil (Callosobruchus chinensis) and cigarette beetle (Lasioderma serricorne) are pests that attack post-harvest crops in warehouses, and book louse (Liposcelis bostrychophilus) cause damage manuscript stacks in museums. ${ }^{22}$ Common clothes moth (Tineola bisselliella), bathroom fly (Clogmia albipunctatus), German cockroach (Blattella germanica), oriental termite (Coptotermes formosanus) are pests resident in homes and offices ${ }^{22}$ and the Asian tiger mosquito (Aedes albopictus) is a human pest. ${ }^{23}$ Insect pests in agricultural and horticultural environments are most suitable targets for our electrostatic approaches. Indeed, our hydroponic tomato culture is conducted year-round in the greenhouses, and tomato plants frequently suffer from pathogen infection and/or insect attack. Infection with insect-carried viruses causes severe damage to tomato plants. Tomato yellow leaf curl virus (TYLCV), carried by the whitefly, has represented the most serious threat in high-temperature seasons. ${ }^{24}$

Cucumber mosaic virus (CMV), carried by the green peach aphid and tomato spotted wilt virus (TSWV), carried by western flower thrips, have also occurred frequently. In addition, shore flies, which inhabit and multiply on alga lawns in sponge cubes soaked with hydroponic culture solution, have been found to transmit rhizosphere pathogens, such as Verticillium wilt, Verticillium dahliae and Fusarium crown and root rot, Fusarium oxysporum f. sp. radicis-lycopersici. ${ }^{25}$ ${ }^{26}$ To minimize the entry of these insect pests to greenhouses, insectexcluding woven screens with a fine mesh size (0.3-mm mesh) have been extensively employed, but the disadvantage of screening is a reduction in ventilation that causes overheating and an increase in relative humidity. Of the small insect pests passing through a conventional insect-proof net (approximately $1.5 \mathrm{~mm}$ mesh), the whitefly is a major pest in tomato cultivation. ${ }^{27}$ As mentioned earlier, the greatest economic threat is due to the transmission of damaging plant viruses, primarily the Geminiviruses. ${ }^{28,29}$ Whitefly has been difficult to control with insecticides because it feeds and oviposits mainly on the abaxial leaf surfaces,$^{30}$ and because it has developed resistance to most classes of insecticides applied for its control. ${ }^{31-34}$ Physical methods could provide an alternative means of managing the pest, since they would be compatible with other components of integrated pest management, have little impact on the environment, and reduce pesticide use, thus slowing the development of insecticide resistance. ${ }^{35}$ Also, in Japan a viral disease caused by Tomato yellow leaf curl virus is vectored by $B$. tabaci and is a major cause of loss of tomato crops grown in greenhouses nationwide. ${ }^{36}$ To solve this problem, we used an electrostatic spore precipitator, which had been developed to control tomato powdery mildew. ${ }^{12}$ This device is so effective in attracting air-borne conidia from the air that tomato plants guarded by the spore precipitator remain uninfected. ${ }^{12}$

Despite the success of an electric field in pathogen control, a preliminary attempt to utilize this device for pest control was unsuccessful, because the electrostatic force of the spore precipitator was insufficient to maintain a hold on trapped whitefly adults. A successful application of the SD-screen was a practical achievement to manage not only whiteflies but also other greenhouse pests. ${ }^{14-38} \mathrm{In}$ the area of postharvest crop protection, two storage insects, cigarette 
beetles and vinegar flies, have been targeted as the most serious pests to be expelled. Adults of the cigarette beetle damage a wide range of stored agricultural products, including cocoa, beans, cereals, cereal products, oilseeds, pulses, spices, dried fruits, cured tobacco leaves, and some animal products. ${ }^{22}$ Both larva and adult red flour beetles damage dry food materials, such as ground rice, wheat flour, cornmeal, spices, cottonseed flour, and livestock foods. Adult rice weevils cause serious damage to rice grains in addition to several cereal grains and beans. These pests can multiply rapidly in storage bags and spoil postharvest crops, and in postharvest crop protection, these insects have been targeted as the most serious pests. ${ }^{22}$ In addition, some small flies, such as the Mediterranean fruit fly ${ }^{39,40}$ and vinegar fly, ${ }^{41}$ have been reported to transfer human-pathogenic bacteria to postharvest wounded fruits. In our attempts to physically trap storage pests with an electrostatic device, we found that insects strongly avoid the electric field of our electrostatic device. This finding pushed us to develop an electric field-forming screen that both repels insects that come close to the electric field and also captures insects that casually enter the electric field. ${ }^{42}$ In addition to this electric field screen research, we proposed a system to monitor the pests in a warehouse and provided some practical measures to solve the pest problem. ${ }^{43}$

Special concern of warehouse pest control is the management of the rice weevil; in fact, the rice weevil is the most destructive pest of grains, seeds, and grain products stored in elevators and bins..$^{22}$ Adult rice weevils live for 4-5 months and each female lays 300-400 eggs during this period. The female uses her strong mandibles to chew a hole in the grain kernel where she deposits a single egg and seals the hole with a gelatinous fluid. Larvae develop within the grain kernels as they eat and frequently cause almost complete grain destruction in storage facilities. The rice weevil is insidious, because adults disperse by flight, allowing infestations to spread to neighboring areas. Prevention of entry is the best strategy to avoid insect infestations in stored grains, ${ }^{42}$ and proper sanitation before the introduction of new grain is essential to minimize the risk of insect contamination of the new grain. New grain should therefore be screened to eliminate fine materials and broken kernels before storage in a bin, and stored grain should be checked at regular intervals. Despite these precautions, however, infestation occurs frequently in many storage facilities, and eventual fumigation of the grain is inevitable. Nevertheless, fumigation is difficult and costly due to the high toxicity of registered fumigants and the technical knowledge required for their proper use. ${ }^{22}$ This problem was finely solved by developing a new electrostatic device to selectively eradicate rice weevils nesting in rice grain. ${ }^{44}$ Mosquito problems are another breakthrough of our electric field screen research. The first problem is the prevention of virus-carrying mosquitoes from escaping a piggery. In the design of modern livestock facilities, it is essential to ensure adequate ventilation. ${ }^{45}$ However, the provision of sufficient ventilation could lead to the introduction of insect pests into the facility. There is a diverse range of serious pest problems in livestock production. Livestock pests feed on the blood, skin, and hair of animals ${ }^{46}$ and their bites can cause physical and mental health issues for the animals. In addition to pest problems in the livestock, there are also concerns regarding the pest-mediated transmission of pathogens from livestock facilities to surrounding human communities. The rapid pace of urbanization in many parts of the world has created the need for urban animal husbandry to supply city residents with food. The potential transmission of emerging zoonotic diseases in urban areas has therefore become increasingly important. Mosquitoes carry many different disease pathogens and viruses that can infect vertebrates. ${ }^{46}$ Our focus is to prevent the mosquito-mediated infection of humans by Japanese encephalitis virus (JEV). JEV is a mosquito-borne, zoonotic flavivirus that causes encephalitis in humans and reproductive disorders in pigs. ${ }^{47}$ Ardeid wading birds are the primary maintenance hosts, pigs are the main amplifying hosts, and Culex mosquitoes are the primary mosquito vectors for JEV. ${ }^{46}$

Virus-infected pigs display no clinical signs except that pregnant sows may abort or have stillborn piglets. ${ }^{48}$ In contrast, the disease is extremely serious in humans, especially children, with severe initial symptoms (fever, headache, and vomiting) followed by changes in the patient's mental status, neurologic symptoms, weakness, movement disorders, and seizures. ${ }^{49}$ Lindahl et al..$^{50}$ reported that the presence of vectors (Culex tritaeniorhynchus) and amplifying hosts (pigs) in urban settings led to the rapid spread of JEV among humans in the neighboring area. Suppression of JEV in humans is generally best achieved through the vaccination of humans or swine, mosquito control measures, or a combination of both strategies. ${ }^{49} \mathrm{An}$ alternative approach to the control of these harmful pests is to prevent their passage into or out of animal husbandry facilities using an electrostatic device. For this purpose, we constructed a novel electrostatic screen to selectively kill insect pests that entered the electric field. ${ }^{51}$ The device was designed to be attachable to openings (e.g., windows) in the building. The second problem is to exclude hematophagous mosquitoes from houses. In tropical and subtropical countries, these noxious mosquitoes transmit various viral and bacterial pathogens and malarial plasmodia that cause severe diseases in humans. The current standard countermeasure is the use of an insecticide-impregnated woven net with larger mesh sizes (mesh diameter $5 \mathrm{~mm}$ ) for increased air-permeability. ${ }^{52}$ However, this method has some potential risks, including the development of insecticide-resistant pests $^{53}$ and continuous contact with exuded neurotoxic insecticides by the users. ${ }^{54}$ Our electrostatic technique created pest-free living spaces in houses by installing the screens on windows because its strong electrostatic force captures insects while allowing good air penetration..$^{55}$

\section{Pollen grains}

Pollinosis is an allergic disease caused by windborne pollen. In Japan, pollen from the Japanese cedar (Cryptomeria japonica), Japanese cypress (Chamaecyparis obtusa) and ragweed (Ambrosia artemisiifolia) are the major pollinosis allergens and are widespread nationwide. According to government research, more than 2.5 million people in Japan are sensitive to these pollen allergens and develop symptoms such as headache, eye congestion and itchiness, repeated sneezing with runny nose, throat irritation and pain, physical weariness, and reduced concentration during the annual pollen season. ${ }^{56}$ Normally, people let fresh air into a room by opening windows. To improve the quality of life of pollinosis sufferers during pollen season, we sought to obtain pollen-free space in homes. To this end, we develop an electrostatic-barrier-forming window prevents pollen grains from entering a house. We originally developed similar barriers to trap insect pests for safe crop production and preservation, ${ }^{14-58}$ and this concept was expanded to a new electrostatic apparatus for capturing windborne pollen grains. ${ }^{59}$ The structure of the proposed apparatus is very simple and easy to construct, and most importantly it can operate continuously for a long period with low energy consumption. 


\section{Smoke particulate matter}

Smoke is made up of a complex mixture of gases and fine particles produced when wood and other organic matter are burned. The biggest health threat from smoke comes from fine particles. These microscopic particles can get into the eyes and respiratory system, where they can cause health problems such as burning eyes, runny nose, and illnesses such as bronchitis. Fine particles can also aggravate chronic heart and lung diseases and are linked to premature death. ${ }^{60}$ Our primary concern was a transboundary air pollution. The pollutants were frequently transmitted to Japan by the prevailing westerlies, because of geographical relation to the air-pollutant generating country. The state and local agencies provide air quality forecasts reporting when particle levels are expected to be unhealthy during regular environmental monitoring of actual levels of common air pollutants. When an alert is issued, we are advised to stay indoors and close windows and doors to keep indoor air as clean as possible until the alert is cleared. Our goal was to improve the quality of life of those sensitive to air pollutants by eliminating airborne particulate matter (PM) in homes. To this end, we developed an electrostatic air purification screen that prevents fine PM in the air from entering the household. ${ }^{61}$ The apparatus for capturing fine, airborne particles is simple and easy to construct; most importantly, the system is energy-efficient and can operate continuously for long periods of time. Passive smoking is the inhalation of environmental tobacco smoke (i.e. of second-hand smoke by persons that do not intend to smoke). Sidestream tobacco smoke permeates the environment, leading to inhalation by people within that environment. Exposure to second-hand tobacco smoke can cause disease, disability, and death. ${ }^{62}$ The health risks of secondhand smoke are a matter of scientific consensus; in fact, second-hand smoke causes many of the same diseases as direct smoking, including cardiovascular diseases, lung cancer, and respiratory diseases. ${ }^{63}$ In particular, pregnant females exposed to environmental tobacco smoke have a higher risk of delivering children with congenital abnormalities, longer lengths, smaller head circumferences, and low birth weights. ${ }^{64}$ In addition, the evidence is sufficient to infer a causal relationship between exposure to second-hand smoke and sudden infant death syndrome..$^{65}$ Thus, second-hand smoke is the most significant indoor pollutant for humans. These risks served as a major motivation for smoke-free laws in workplaces and indoor public places. Here, we attempted to physically eliminate tobacco smoke, to keep indoor air as clean as possible, by developing an electrostatic air purification device that prevents tobacco sidestream smoke from entering public spaces and thus affecting non-smokers.

\section{Construction of discharge-free electric field screens}

The electric field screen is the most basic and important device for creating spore-free and pest-free spaces for agricultural crops and their products for safe cultivation, processing, and preservation ${ }^{3}$ For this purpose, a discharge-free type of the screen has been firstly developed to prevent the entry of airborne conidia of plant pathogenic fungi and small, flying insect pests that permeate a conventional woven insect net and yet ensure good air penetration (Figure 1A). Successful exclusion of pathogens and pests could be achieved by trapping targets that get inside the screen or by repelling the pests reaching the outside net of the screen. As shown in Figures 1B-F, various types of electric field screen were developed during the investigation. These screens are single-charged monopolar electric field screen (SM-screen) (Figure 1B), ${ }^{12,} 66$ single-charged dipolar electric field screen (SDscreen) (Figure 1C), ${ }^{14-73}$ double-charged dipolar electric field screen (DD-screen) (Figure 1D-F). ${ }^{17-61}$ In addition to these screens, discharge generating electric field screens ${ }^{44-74}$ are described with different aims of protection. The detailed principles, functions, and applications of individual electric field screens are discussed in the following sections, and their application to the sites of different environments is described as representative examples of the screen installation. A greenhouse is a specific environment in which we firstly intended to create an airy condition that successfully excludes insect vectors (whiteflies, green peach aphids, western flower thrips and shore flies) of pathogens and airborne conidia of powdery mildew pathogens for healthy crop production. In this review article, we describe different types of electric field screens and their related devices as the remarkable achievement of being the newly devised electrostatic instruments.

A
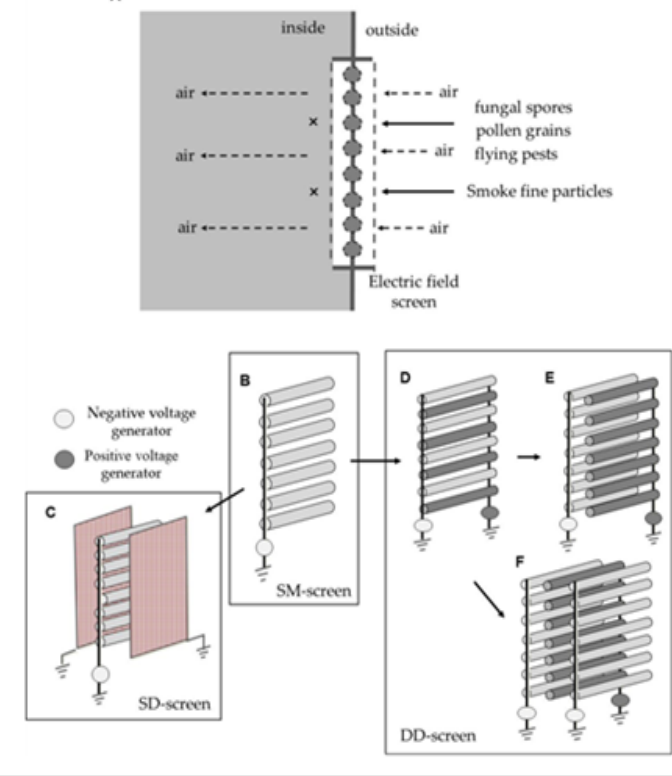

Figure I Conceptual diagram for the roles of the electric field screen (A) and development of various types of electric field screen (B-F). Single-charged monopolar electric field screen (SM-screen) is the original type for singlecharged (SD-screen) and double-charged dipolar electric field screen (DDscreen).

\section{Electrostatic spore collection probe; an original invention leading to electric field screen research}

The trigger of our continued electrostatic research is the finding that single spores of the powdery mildew pathogen colonizing on host tomato leaves could be collected with a fine needle that was made by drawing a heated glass pipette. This finding was brought about during a micromanipulation of a glass needle conducted under a digital microscope to collect living mature conidia formed on a target conidiophore on a plant leaf without causing any detrimental effect on their survival, aiming at analysing gene expression in the targeted single conidia. ${ }^{75}$ Interestingly, conidia jumped up to the glass needle before touching when the needle was brought close to the conidiophore. Obviously, this interesting phenomenon indicates the involvement of an electrostatic attraction force between the spore and the glass needle. The vital point of this phenomenon is the use of the drawn glass needle. The glass needle was made by heating a glass pipe at a given point, stretching it mechanically, and cutting it at an arbitrary point. In our interpretation, the stretching is very important; the surface of the glass-pipette was electrified by triboelectricity 
(frictional electricity) produced by the friction between the glass surface and the surrounding air (triboelectrification) ${ }^{76,77}$ Electricity accumulated on the glass surface localizes at the tip portion when the glass-pipette is cut, because the electricity has a nature to get together at the edge surface. Finally, the electricity produces the electrostatic field in the space around the pipette. The electrostatic field (electric field) generates the force of spore attraction. To evaluate the feasibility of our interpretation, an electrostatic spore collection probe was constructed to actually collect mature conidia on conidiophores, using the force of the electric field. ${ }^{78,79}$ The probe is a pointed ebonite (insulator), and its pointed end is electrified in a dielectric polarization of the ebonite when its opposite end was touched with an aluminum cap (conductor) negatively charged with a voltage generator. The electric field is produced in the space around the pointed tip. ${ }^{79}, 80$ Our study showed successful electrostatic attraction of conidia to the probe tip; the works of electrostatic spore collection were published in some journals. ${ }^{81-83}$ This finding was the start of our electric filed screen research. Hopefully, this force is available to the physical barrier for trapping air-borne conidia of fungal pathogens.

\section{Basic system for voltage generation and electricity supply}

The initial approach in the electric field screen research is to use electrostatic forces to trap wind-carried fungal spores and flying pests. The vital point of the research is to consider how to create the electrostatic force and then how to make the force strong enough to capture all targets. The first problem can be solved by the use of an electrified conductor (a material that conducts or transmits electricity). The conductor receives electricity in accordance with its own electrostatic capacity and potential difference against an electricity donor. The electricity accumulates in the surface of the conductor and generates an electric field in the space contiguous to the electrified conductor. The expanse of the electric field is directly proportional to an increase in the amount of electricity accumulated on the conductor. In the electric field, the electricity-accumulating conductor acts as a negative pole and creates an opposite pole by polarizing a material placed in the electric field; a conductor is polarized as a result of electrostatic induction, ${ }^{78}$ and an insulator (a substance that does not readily conduct electricity), polarized by dielectric polarization. ${ }^{78,79}$ An attractive force is generated between the opposite poles. The strength of the attractive force depends on the distance between the poles; the force becomes stronger as the distance decreases.

A direct current (DC) voltage generator (amplifier) is a booster in which a set-up potential transformer and a Cockcroft circuit are used to enhance an initial voltage from a storage battery $(12 \mathrm{~V})$ to desired secondary voltages $(0.2-30 \mathrm{kV})$. The electric power consumption of the voltage generator is $0.5 \mathrm{~W}$, equivalents to a small electric bulb, and therefore the voltage generator can be operated by a $12-\mathrm{V}$ storage battery. The battery has power supplied through photovoltaic power generation by a small solar panel, of size $10-15 \mathrm{~cm}$. The enhanced voltage of the generator is used to pump electricity from ground and to supply it to the metal wire conductors. One of the most important points in the construction of the electric field screen is the insulation of the conductor wire (copper or iron wire) with an acrylic cylinder or a vinyl chloride sleeve. In non-insulated conductors, the electricity accumulated on the conductor surface moves towards another conductor when it approaches a charged conductor: that is, when the second conductor enters the electric field produced by the first conductor. ${ }^{78}$ This electricity transfer is recognized as arc discharge in many cases. ${ }^{84}$ The human body can act as a conductor and can receive electricity from the charged conductor when we enter an electric field (an electric shock or electrocution). The intensity of the electric shock depends on the amount of electricity on the electrified conductor (donor conductor), the capacitance of the human body (recipient conductor), and the potential difference between the donor and recipient conductors. The use of non-insulated conductors would expose the screen user to danger of electric shock; in fact, a large electric shock may cause serious harm to users.

Insulating the conductor wires is a fundamental safeguard of the electric field screen that suppresses potentially dangerous discharges from the charged conductor. The ability of insulators to prevent the passage of electricity is expressed as volume resistivity; generally, materials with more than $10^{5} \Omega \cdot \mathrm{cm}$ are regarded as insulators. Charging a conductor beyond the maximum permissible limit causes a leak of excess electricity through the cover of the insulator; that is, there is a reduction of its insulation resistance.$^{80}$ The surface potential of the charged conductor is measureable using a field meter and consistent with the voltage produced by the voltage generator. In an actual approach, we can determine the upper limit of conductor-charging by changing the voltages applied and by measuring the occurrence of an electric current from the insulator sleeve surface. Another important point in charging an insulated conductor wire (ICW) is the specific manner of the polarization of insulator materials. Namely, dielectrically polarized insulator sleeve coats the conductor wire; the sleeve is negatively charged on the outer surface and positively on the inner conductor wire surface. This polarization is generated as a result of the regular orientation of dipolar molecules involved in an insulator; the opposite charges of inner molecules neutralize each other, and eventually the outside molecules of the insulator remain negatively or positively charged in an electric field. ${ }^{78,79}$ The surface potential of the dielectrically polarized insulator corresponds to the potential of the charged conductor wire, and therefore this potential is also freely changeable by altering the voltage of the voltage generator.

\section{SM- screen}

The negative charge on the surface of a dielectrically polarized insulator produces an electric field in the surrounding area. In this electric field, the expanse and strength of the field is in direct proportion to the amount of electricity supplied to the conductor; that is, the voltage amplified by the voltage generator. We use the insulator sleeve that adheres closely to the central conductor wire. However, this situation is not essential to polarize an insulator cover. This is merely a case; polarization occurs at any time when an insulator is placed in an electric field. Alternatively, an acrylic cylinder is used for the insulation of the conductor. In this configuration, there is a space between the conductor wire and the cover insulator cylinder. The negatively charged conductor produces an electric field in the inner space to polarize the cylinder dielectrically. The negative surface charge of the cylinder produces the second electric field in the space around it. The point of interest is the reduction of the potential on the insulator surface, because the degree of the polarization is determined by the field strength at the point of the electric field where the cylinder is positioned. The field strength becomes weaker as the distance from the charged conductor increases. The main discussion point of this section is the creation of an electrostatic force that attracts airborne spores entering the outer electric field. The most reasonable explanation is dielectrophoretic movement of the spores subjected to a non-uniform electric field. Dielectrophoresis is a phenomenon in which a force is exerted on a dielectric particle (oppositely polarized particle) in a non-uniform electric field. ${ }^{85}$ This force does not require the particle to be charged, because all particles 
exhibit dielectrophoretic activity in the presence of a non-uniform electric field. Obviously, with the round-shaped electrodes used in the electric field screen, it is possible to produce a non-uniform electric field so that spores could be polarized dielectrically. According to the dielectrophoresis theory, the relative polarizability of the spores to the surrounding electric field changes along the gradient of the electric field strength. This changeable polarization enables the spores to move towards the electrodes. Finding this dielectrophoretic attraction of spores in the electric field was our starting point in developing the idea of an electrostatic space barrier through which airborne spores are prevented from passing.

In our practical approach, ${ }^{12-66}$ the conductor-covering cylinders are arrayed in parallel and at constant intervals to form a vertical layer of the cylinders. The point in this construction is to create no space between the electric fields to prevent passage of wind-carried spores through the electrostatic barrier. As mentioned earlier, the electric field is formed concentrically around the charged conductor, and the expanse of the field becomes wider with an increase in the voltage applied. That is, the interval between the cylinders can be widened to enhance the air permeability of the screen, if we apply higher voltages. From the practical view point, however, larger voltages increase the danger of discharge at contact points in the electric wiring among the cylinders and between the cylinders and the voltage generator; naturally, higher costs and techniques are required to control the occurrence of discharges, which may otherwise lead to unexpected accidents. It is more realistic to use lower voltages, and to make the interval between the cylinders narrower, with the condition that the minimum necessary air permeability can be ensured. This is the story for the birth of the SM-screen.

\section{SD-screen}

The second invention in our electric field screen research is the formation of a dipolar electric field. In the process leading to this, however, several technical problems stood in our way. One of the most important problems is the difficulty in processing an acrylic resin (used for insulating the charged conductor) into the various shapes required for constructing new types of electric field screen. Consequently, we adopted a soft vinyl chloride as the second insulator material, because of its easy processing and wide availability in commercial materials. However, the use of a softened vinyl chloride sleeve raises an additional problem; the reduced resistivity versus the original insulator material caused by the addition of plasticisers with higher conductivities. As a result, the applicable voltage becomes lower $(<10 \mathrm{kV})$, and therefore the attractive force of the screen becomes weaker. This situation is more important when we attempted to trap insect pests. In fact, with the conductor wire insulated with a vinyl chloride sleeve, it was possible to attract the insects that entered the electric field to the insulated conductor, but the force was insufficient to restrain them; insects bent their head and/or tail upwards and twisted their bodies as they were lying on their backs and finally flew away from the screen. To address this, we developed a new system for forming a dipolar electric field. In this system, an earthed metal net (conductor) is placed inside the electric field to make the opposite pole. As a result of electrostatic induction in the conductor net, its inside surface is polarized positively; free electrons are pushed out of the net towards a ground so that the net surface becomes positive. In this configuration, the insulated conductor wire and the earthed metal net become the negative and positive poles, respectively, and the cover sleeve and the air between the poles acted as a resistance against the flow of electricity from the conductor wire to the metal net. Within the permissible voltage range, a static electric field can be maintained between the poles. Naturally, the field strength is determined by the distance between the poles, air conductivity, and the voltages applied.

The vital point of our invention is the movement of external electricity that enters this field. This field exerts an electrostatic force to push external electricity out of the field to ground, via the metal net. In concrete terms, insects are deprived of free electrons from their outer surface cuticle layer when they enter the electric field. As a result of electron deprivation, the insects are positively electrified and strongly attracted to the negative pole trap. In fact, the force is so strong that the insects cannot escape this electrostatic trap. Obviously, the critical phase in this attraction mechanism is the positive electrification of the insect in the dipolar electric field, designated as biological discharge for positive electrification. ${ }^{67}$ The level of insect electrification is dependent on the surface potential of the insulated charged conductor. The SD-screen is constructed according to the following procedure. The vinyl chloride sleeve-covered conductor wires are arrayed in parallel and at constant intervals $(5 \mathrm{~mm})$, and then two earthed stainless nets are placed on both sides of the conductor wire layer. Then, the voltage applied to the generator is raised without changing the interval between the insulated conductor wires; alternatively the interval is shortened without changing the applied voltage. By these treatments, the neighbouring electric fields exerted a repulsive force against each other that was elliptical with longer flat borders. This treatment is effective in increasing the area of the electric fields so that the side surface of the net is entirely electrified. The SD-screen is able to tightly capture all insect pests that enter the electric field inside the screen. In fact, all tested insects passing through the screen net (mesh diameter, $1.6 \mathrm{~mm}$; equivalent to the mesh size of a conventional woven insect net) were trapped by the charged insulated conductor wires. Tested insects include greenhouse pests (whiteflies, western flower thrips, green peach aphids, tomato leaf minor flies and shore flies), warehouse pests (cigarette beetles, red flour beetle, Azuki bean weevils, and rice weevils), food processing factory pests (vinegar flies and humpback flies), and house and museum pests (German cockroaches, Asian tiger mosquitoes, Oriental termites, and book louses) ${ }^{68}$ By charging the conductor wires to $4.2 \mathrm{kV}$, the capturing force is sufficiently strong that trapped insects are not released from the trap even when blown at $7 \mathrm{~m} / \mathrm{s}$.

\section{DD-screen}

Although the SD-screen is sufficient to trap insects entering the screen, its weak point is that there is no static electric field between the conductor wires. Successful insect capture depends on the formation of an electrostatic barrier with no spaces through which the insects can pass. However, parallel conductor wires with negative charges create an electric field (electrostatic field) with no electricity transfers between the conductors or insects, which is ineffective at attracting insects into the field. In a survey of screens installed on greenhouse windows, we observed that insects rarely entered this field. To overcome this, we proposed a new electric field screen with oppositely charged conductor wires of equal voltages using two voltage generators. The third electric field screen (DD-screen) forms gap-free electric fields with no spaces for insect escape.$^{57}$ In this configuration, a static electric field is generated between the charged insulated conductor wire and the earthed net and between the oppositely charged conductor wires. A unique feature of this type of electric field screen is the locally different polarization of the uninterrupted earthed net; a net part facing an electric filed produced 
by the negatively charged conductor wire polarizes positively, and another net part facing an electric field by the positively charged conductor wire polarizes negatively. This alternative polarization causes different electrification of the insect, depending on the electric field of the same electric field screen where the insect enters.

Three types of an electric field (fields ' $a$ ' to 'c') are produced inside the DD-screen. Field ' $a$ ' is identical to the electric field of the SDscreen, causing biological discharging for positive electrification of an insect. In contrast, in field ' $b$ '. the positive charge on the insulated conductor wire draws electricity from ground to accumulate it on the field-side surface of the earthed net. This electricity is transferred to an insect (biological charging for negative electrification). These two electrifications are detected as transient electric currents in reciprocal directions by a galvanometer integrated in the earth line of the metal net. This transient current occurs immediately after an insect is released in the alternative electric fields ' $a$ ' or ' $b$ '. If we simultaneously release two insects in both fields, the electric current becomes zero because of opposite flows of electricity in the same earthed line. In both fields, insect electrification is the trigger of the subsequent attraction to the charged insulated conductor wires, because the attractive force is generated between the opposite charges of the insect and the conductor wire. Also, in field 'c', the insect electrification is an initial and essential event for trapping insects that enter the electric field. Biological discharge (positive electrification of insects) occurs immediately after the insect release into the field. Negative charge pushes out of the insect and accumulates in the space around the positively charged conductor wire, because an insulator sleeve covering the conductor is sufficiently resistant so that insectderived negative charges cannot move to ground via the metal net. It is advantageous for field ' $c$ ' to create a field strength stronger than those of the other two fields; in the present case, the potential difference ( 8.4 $\mathrm{kV}$ ) between the oppositely charged conductor wires is twofold higher than those between the positively or negatively charged conductor wire and the earthed net in the fields ' $a$ ' and ' $b$ ' ( $4.2 \mathrm{kV}$ in each). This enables forming the strongest barrier of the electric fields vertically at the central position of the electric field screen. The DD-screen is the ultimate form in terms of the strongest power of insect capturing.

\section{Non-earthed electrostatic circuit for screen electrification}

From an electrostatic point of view, the present screen is regarded as an apparatus to use an electrodynamic phenomenon mediated by static electricity. As mentioned previously, in an ordinary electric field screen, the high voltage (secondary voltage), boosted by the voltage generator, is used to draw electricity from a ground and confine it in an insulated conductor wire for making static electricity. This static electricity pushes an equivalent free electron from the metal net to ground. This is an 'earth-to-earth' circuit involving a static electric field, designated an earthed electrostatic circuit. However, the invention described in this section is a non-earthed electrostatic circuit in which two earth lines from the voltage generator and metal net are linked to each other. In this circuit, the free electrons of the metal net are the source of the electricity that accumulates in the insulated conductor wires; this electricity movement causes an opposite polarization of the insulated conductor wires and metal net, creating two opposite poles. Applying an equal voltage, the strength of the static electric field between the opposite poles is identical in both earthed and non-earthed electrostatic circuits. The non-earthed electrostatic circuit is also integrated in the DD-screen. ${ }^{55}$
In this type, the electricity (free electrons) of the one-side conductor wires is drawn by the voltage of the positive voltage generator and furthermore pushed towards the other-side conductor wires by the equal voltage of the negative voltage generator. Accordingly, the potential difference between the poles is duplicated, if the pole-topole distance is the same as that of the SD-screen, and therefore the DD-screen can exert a strong force in a static electric field even if the non-earthed circuit is used. The invention of the non-earthed electrostatic circuit is the latest contribution to moving electric field screens towards practical use. Before developing this circuit, it was necessary to connect the earth lead of the electric field screen to the terminal, and therefore the establishment of the earth terminal was a prerequisite for use of the electric field screen in different locations with different electric facilities. Obviously, this time- and costintensive process limits the popularization of the use of the electric field screen among the general public. The problem is most serious when an electrostatic crop reservoir (a type of mobile container installed with the electric field screen) is used in a warehouse where the floor is coated with insulator flooring materials. The reservoir is effective to create an airy but pest-free space inside the container and useful for a warehouse where the entrance door is frequently opened and closed, because such a warehouse is exposed to a high risk of pest entrance. The problems we faced were to ensure the contact of the screen terminals (earth lines of the voltage generator and the metal net) with the ground. In addition, an insulated floor surface is a barrier to successful earthing. The use of the non-earthed electrostatic circuit solves this problem. This circuit is applicable to all types of dischargefree electric field screen.

\section{Insect-repelling function of the discharge-free dipolar electric field screens}

The third invention in our electric field screen research resulted from the finding that insects are deterred from entering the inner space of dipolar electric field screens. Importantly, this was common to a broad range of insect species. ${ }^{69}$ Our assay covered insects extending over 17 orders, 42 families, 45 genera, and 82 species. As far as we are aware, no insect tested flew directly through the screen net. The insects approached the dipolar electric field screen, perched on the string of the outside net surface, and then inserted their antennae inside the screen before entering. This behaviour was common in all tested insects and was observed even when the screen was not charged. Obviously, the insects search the interior with their antennae before entering. At optimal voltages for insect capture (1.2-4.2 kV, depending on the sizes of the targeted insects), almost all insects flew away from the screen immediately after reaching the screen net. In rare cases, however, the insects are drawn inside the screen when they lose their balance on the screen net, especially when they try to insert their antennae inside, because the attractive force of this voltage application is very strong. In fact, insects recognized an existence of the dipolar electric field even if the screen was set at much lower voltages; all insects tested sensed and avoided the field at $>0.8 \mathrm{kV}$. This finding was a turning point in changing our thinking in the use of the electric field screen.

The electric field screen possesses two functions: i) an insectcapturing ability, based on its own electrostatic features, and ii) an insect-repelling ability, based on the common habit (dipolar electric field avoidance) of insects. Especially, the second of these is important as a basic element to formulate an efficient and realistic strategy for pest management, because our intensive survey indicated that the 
dipolar electric field screen repelled all insects reaching the screen net. The aim of using the electric field screen is to exclude pathogens and pests from the places intended to be protected (greenhouses, warehouses, food processing factories, animal husbandry facilities, and houses). To achieve this purpose effectively, the repelling-based and capturing-based electric field screens were designed, and the 'right' type of electric field screen was used for the 'right' type of protection. In fact, the electric field screens with the earthed net (SDand DD-screens) were classified as repelling-based screens. However, these repelling-based screens are readily changed to the capturingbased type merely by detaching the earth line from the metal net of one side or on both sides. The screen conversion is explained using the double-charged dipolar electric field screen, because of the static electric field generated in the space between the oppositely charged insulated conductor wires. This space is the field to capture insects, but the capturing force was sufficiently strong to trap all of the major pests we tested. By removing the earth line, insects that reach the nonearthed net of the modified screen rapidly enter the screen without hesitation regardless of the applied voltage and are tightly captured by the insulated charged conductor wire. The earth line on one side is removed to construct an electric field screen with different functions on both sides. Naturally, in removing the earth lines on both sides, the screen exhibits an insect-capturing function. In our pest management strategy, the repelling-based screen is the forefront barrier that prevents invasion by outdoor pests, while the capture-based type is the inner trap for pests that manage to enter facilities. ${ }^{58}$

\section{Exemplification of electric field screen application}

\section{Electric field screen-guarded greenhouse}

The first application is the installation of the electric field screens to a greenhouse to keep the inside spore-free and pest free. ${ }^{58}$ For this purpose, two types of the screen, SD-screen (Figure 1C) and DDscreen (Figure 1D) are used to repel the insects reaching the screen net installed to the lateral windows and to directly capture the insects through the entrance door of the greenhouse, respectively. Figure 2A shows a A-shaped greenhouse whose lateral windows are furnished with SD-screens attached to the window frame. All screens are linked in the non-earthed electrostatic circuit and operated by one small storage battery powered by a small solar panel. A small pre-entrance room is constructed inside a greenhouse next to the entrance door. This is a room to trap insects entering through the door. This room is furnished with three types of DD-screen; the yellow- and grayboarded DD-screens are fitted in the central wall side and the inner door of the room, respectively, and the gray-netted DD-screen is integrated into the wall opposite the inner door. In this configuration, these screens are installed in the pre-entrance room taking into consideration the airflow inside the room, as most insects are brought in by the air when the door is opened. Two DD-screens with the board are useful for directing the wind toward the wall into which the netted DD-screen is integrated. Insects are eliminated by this screen, and the pest-free air is circulated inside the greenhouse. The yellowboarded DD-screen is highly attractive because of the photo-selective movement of the whiteflies and is effective for trapping the whiteflies when there was no wind. Our electrostatic method was effective at keeping the greenhouse pest-free throughout the entire period of tomato cultivation. ${ }^{58}$ Incidentally, roof windows are not furnished with the screens because of the structural difficulty of doing so, and these remained closed during the experiment. Extractor fans are fitted on the front wall of the greenhouse. The extractor fan is covered with a box of SD-screens to prevent the entry of insects through the gap of the fan. An air-circulating fan is attached to a crossbeam in the room, and all fans begin operating automatically when the inside temperature reaches $30^{\circ} \mathrm{C}$. All side windows are automatically closed when the outside air speed reaches $3 \mathrm{~m} \mathrm{~s}^{-1}$ and are opened again when the wind decreased to $1.5 \mathrm{~m} \mathrm{~s}^{-1}$.

\section{Bamboo blind-type electric field screen}

Crop plants in our greenhouse cultivation system are grown in a high-tech glasshouse with the windows and entrances guarded by an electric field screen to prevent the ingress of airborne pathogens and flying insect pests. ${ }^{12-} 37$ This system is effective for excluding major pests from the greenhouse, including whiteflies, western flower thrips, green peach aphids, and tomato leaf-miner flies, ${ }^{21}$ as well as conidia of tomato powdery mildew. ${ }^{4-11}$ Thus, our electrostatic exclusion method helps to create a pest- and pathogen-free space inside the greenhouse. However, the equipment for this system is very expensive, such that installing the electric field screen would likely be prohibitively expensive for small farmers. To provide a more costeffective alternative method, we proposed a simple bamboo blindtype electric field screen (Bb-EFS), which is suitable for the openings of a cold-frame plastic hoop greenhouse (Figure $2 \mathrm{~B}$ ).$^{73}$ The Bb-EFS is constructed using an insulated conductor wire (ICW); an iron conductor wire (2-mm diameter, 1-m length) is insulated by passing it through a transparent insulator vinyl sleeve (1-mm thickness, $1 \times$ $\left.10^{9} \Omega \cdot \mathrm{cm}\right)$. The Bb-EFS has two components: a layer of ICW and two electrostatic direct current voltage generators. The ICWs are positioned parallel at a 4-mm interval using polypropylene rod spacers (insulator) (4-mm diameter, 1-cm length), which are connected to each other and to a negative or positive voltage generator. The generators are operated to supply equal negative and positive voltages to the ICWs. The two voltage generators are operated using $12 \mathrm{~V}$ storage batteries, with power supplied by a $15 \mathrm{~W}$ solar panel. In this system, free electrons from ICW(+) are pushed out to $\operatorname{ICW}(-)$ (non-earthed electrostatic circuit). Cover sleeves are dielectrically polarized, positively on the surface of the iron wire side and negatively on the outer surface of the insulator sleeve in the $\operatorname{ICW}(-)$, and vice versa in the ICW $(+) .{ }^{57}$ The opposite surface charges on the ICWs act as dipoles that create an electric field between them. The ability of the $\mathrm{Bb}-\mathrm{EFS}$ to trap greenhouse pests was effective enough to minimize their population in the greenhouse. ${ }^{73}$

\section{Electrostatically guarded nursery cabinet and seedling shelter}

The second application is a construction of an electrostatic nursery cabinet (ENC) (Figure 2C) ) $^{38}$ and electrostatic seedling shelter (ESS) (Figure 2D-Figure 2E), ${ }^{12-15}$ which are placed in a non-guarded greenhouse. The ENC is a rectangular cabin whose three lateral walls are furnished with DD-screens (Figure 1E) and ceiling is covered with a transparent vinyl sheet (Figure 2C). The electric field screen used in the ENC has two components: two layers of the ICWs in parallel arrays and two DC voltage generators that supply negative and positive voltages to the ICWs, respectively. The ICWs of each layer are paralleled at a 5-mm interval and connected to each other and to a negative or positive voltage generator. Two ICW-layers are paralleled at a 2-mm interval, and the ICWs of the layers are offset to each other. The ICWs of both layers are oppositely charged with equal voltages. The generators are operated to supply equal negative and positive voltages to the ICWs (the negatively and positively charged ICWs are hereafter represented as ICW (-) and ICW (+), 
respectively). Two layers of the ICWs are integrated in the aluminum frame, and two voltage generators are operated using $12 \mathrm{~V}$ storage batteries, with the power supplied by a $15 \mathrm{~W}$ solar panel. The ESS is fabricated in the form of an electric-field-screened box furnished with three-layered DD-screens (3L-DD screens) (Figure 1F) and placed on a steel worktable (Figure 2D). ${ }^{15}$ Hinged double-doors are attached to the two shorter lateral faces of the box, and the top surface is covered with a transparent polyvinyl film. Three 3L-DD screens are installed on each of the longer lateral faces of the box. The screen has two components: three layers of ICWs in parallel arrays and two electrostatic direct current voltage sources which supply negative and positive voltages to the ICWs, respectively. The ICWs of each layer are placed in parallel with a separation of $5 \mathrm{~mm}$, and are connected to either a negative or positive voltage source; namely, the screen consists of one $\mathrm{ICW}(+)$ layer with two $\mathrm{ICW}(-)$ layers in a parallel array at 2-mm intervals. The ICWs in the different layers are offset from each other. The voltage sources are linked to create an electric circuit producing an electric field between the ICW $(-)$ and ICW $(+)$ insulated wires. Both voltage sources are operated using $12-\mathrm{V}$ storage batteries, with the power supplied by a $15-\mathrm{W}$ solar panel to supply equal negative and positive voltages $(0.4-1.4 \mathrm{kV})$ to the ICWs. In this system, free electrons from ICW (+) are pushed out to the ICW $(-)$ (non-earthed circuit, described in the following section), and the opposite surface charges on the ICWs act as dipoles that form an electric field between them. Another type of the ESS is the simplest version using SM-screen (Figure 1B), which was firstly invented in the electric field screen research to prevent airborne conidia of the powdery mildew pathogen from entering the seedling shelter (Figure 2E) ${ }^{12}$ In this screen, a copper wire (2-mm in diameter) is insulated by passing through a transparent acrylic cylinder with insulating silicon stoppers at the middle and both ends of the cylinder. The cylinders are linked in parallel, and the wire end is connected to the electrostatic voltage generator. The wire is negatively charged by the generator, and the electrostatic field created by the charged wire dielectrically polarizes the cylinder (positive on the inner surface, negative on the outer surface). The electrostatic force produced by the dielectrically polarized cylinder is used to attract the conidia in the air. The hydroponic culture trough is covered with the cubic frame having a total of 120 spore precipitation cylinders $(60-\mathrm{mm}$ cylinder interval; $30 \mathrm{kV}$ applied voltage) to construct an electrostatic seedling shelter.

\section{Related devices of discharge-free electric field screen}

\section{Electrostatic insect sweeper}

In our pest control strategy, the window-installed electric field screen is a basic tool for pest exclusion and can be used in combination with supplementary crop protection methods. One such approach is the development and application of an electrostatic insect sweeper (EIS) (Figure 2F).$^{71}$ The sweeper is a portable, rod-shape apparatus that enables capture of pest insects on leaves by means of an electrostatic force generated in the same manner as the SD-screen. An iron conductor wire (50-cm length, 2- $\mathrm{mm}$ diameter) is passed through a polyvinyl chloride (PVC) sleeve (1-mm thickness) to make the ICW. Eight ICWs are held at constant intervals on the outside of a rigid PVC pipe (3-cm diameter) by double-sided adhesive tape wound along the pipe, and then passed through a cylindrical stainless net $\left(2.5-\mathrm{mm}\right.$ mesh size, $\left.40 \times 10 \mathrm{~cm}^{2}\right)$. The distance between the ICW and net is fixed at $3 \mathrm{~mm}$ by placing a spacer (silicone strip with the same thickness) between both. The ICWs are linked to each other and to the negative terminal, and the net is wired to the positive terminal of a DC voltage generator that is placed inside a grip. Both ends of the ICWs and the net are sealed with silicone cups. The voltage generator is operated with two $1.5-\mathrm{V}$ alkaline batteries connected in series inside the grip. The ICW is negatively charged to dielectrically polarize a cover insulator: positively on the iron wire-side surface and negatively on the outer surface of the insulator sleeve. The negative surface charge of the ICW polarizes the earthed net to create a positive charge on the ICW side surface as a result of electrostatic induction. ${ }^{78}$ An electric field forms between the opposite charges of the ICW and the earthed net. In measuring a mechanical discharge (an occurrence of electric current from the ICW to the net), a galvanometer is integrated into a line between the net and voltage generator. For trapping insects on leaves, the EIS is gently slid along the leaves to capture the insects.

The structure of the EIS is similar to that of the previous screen, ${ }^{42}$ although it is modified slightly to improve its portability. In the original screen, the ICWs possess earthed metal nets on both sides, and high voltages produced through a Cockcroft circuit ${ }^{86}$ of a voltage generator are used to create opposite electrodes; ICWs are negatively charged by the addition of electricity drawn from ground, and the nets are positively charged by pushing electricity (free electrons) of the net to ground. ${ }^{42}$ In the current device, the voltages are used to transfer electricity of the net to the ICWs. In this manner, the ICWs and the net become opposite electrodes and an electric field is formed between them. The most important point of this modification is the removal of the earthed lines from both the voltage generator and metal net (non-earthed electrostatic circuit), which renders the apparatus portable. The flow of the electricity accumulated in ICW depends on the voltage applied to the electrodes, the electrode distance and the insulation resistance of the ICW cover. An electric current from an insulated conductor depends on the insulation resistance at a given voltage, which determines the level of insulator conductivity. ${ }^{80}$ The current is inversely proportional to increases in distance. The EIS was developed as a physical method to supplement a pest-exclusion technique for preventing pests from entering a greenhouse with an electric field screen installed on its lateral windows. The EIS possesses a similar structure as the single-charged dipolar electric field screen, and thus captures insects in the same manner. The EIS is portable and convenient to operate on-site in a greenhouse and is used to eliminate insects from crop plants, as detected during the routine care of plants in the screen-guarded greenhouse. Insects on the plants are attracted inside a sweeper that is gently slid along the leaves. Using this apparatus, all insects invading from the non-guarded entrance door are captured.

\section{Electrostatic flying insect catcher}

A racket-shaped electrostatic flying insect catcher (REIC) is a device to directly remove insects colonizing tomato plants in greenhouses to reduce the population size of these pests, thus reducing subsequent multiplication through their oviposition in the greenhouse. ${ }^{72}$ This apparatus is portable and easy to operate on-site in the greenhouse at the time of daily care of plants. Iron conductor wire is similarly insulated by passing it through a transparent insulator vinyl sleeve and is used to construct the REIC (Figure 2G). Electrical components of the REIC included two layers of insulated iron conductor wires (ICWs) in parallel arrays and two electrostatic direct current voltage generators that supply negative and positive voltages to the ICWs. The ICWs of each layer are arranged in parallel at $5-\mathrm{mm}$ intervals and connected to each other and to a negative or positive voltage generator. The two ICW layers are arranged in parallel with a 2-mm separation between the layers, and the positions of the ICWs of each layer were offset between the layers. The ICWs of both layers are oppositely charged with equal voltages. The generators are operated with $12 \mathrm{~V}$ storage batteries to supply equal negative and positive 
voltages to the ICW(-) and ICW(+), respectively. In this system, free electrons from $\mathrm{ICW}(+)$ are conducted to $\mathrm{ICW}(-)$. Cover sleeves are dielectrically polarized positively on the surface of the iron wire side and negatively on the outer surface of the insulator sleeve in ICW(-) and vice versa in $\mathrm{ICW}(+) .{ }^{57}$ The opposing surface charges on the ICWs act as dipoles that form an electric field in-between. The two layers of ICWs are integrated in the frame of the racket surface (table tennis racket size) and two voltage generators together with a storage battery are put inside the hand grip. The insects flying over the plants are captured by waving the REIC once or twice above the plants. The structure of the REIC is simple; no special technique is required for its construction. The REIC can operate normally at low electric power consumption to capture flying insect pests that cause severe damage in crops. This work demonstrated that the proposed REIC can easily eliminate insects colonizing greenhouse plants leading to healthy growth of the plants to the final stage of fruit production in an openwindow greenhouse.

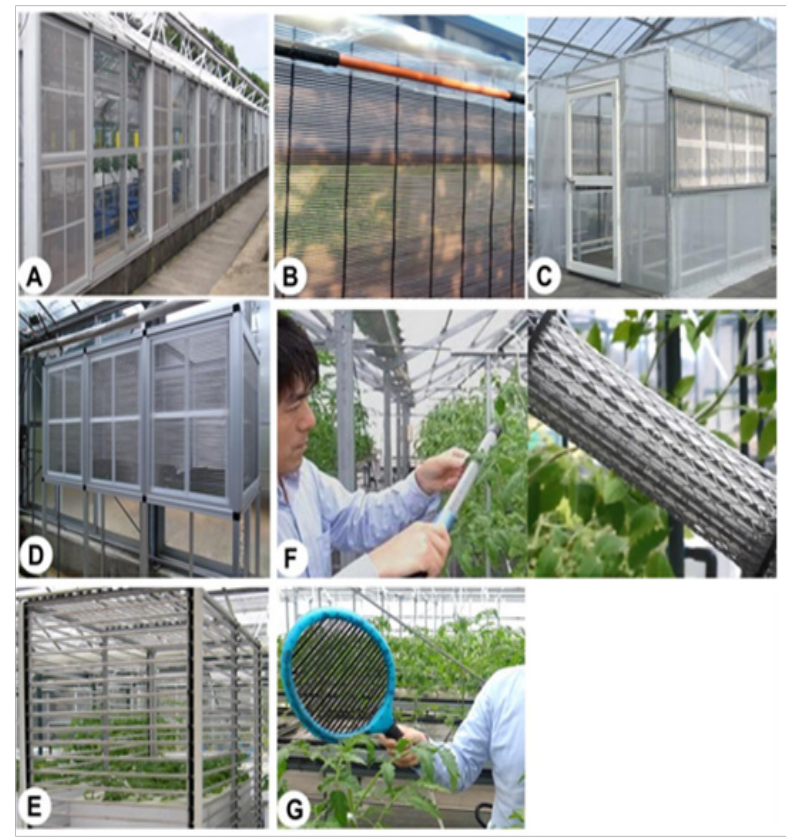

Figure $2 \mathrm{~A}$ greenhouse whose lateral windows are furnished with SDscreens (A), a bamboo blind-type electric field screen (B), an electrostatic nursery cabinet (ENC) (C), an electrostatic seedling shelters (D, E), an electrostatic insect sweeper (EIS) (E), and an electrostatic flying insect catcher $(F)$.

\section{Electrostatic pest-monitoring apparatus}

An electrostatic insect-monitoring system is a device for monitoring the insect pests prowling in a warehouse. ${ }^{43}$ Our experimental design exploits the habitual behavior of insects to enter narrow gaps $\mathrm{s}^{87,88}$ and strips the free electrons from the insect cuticle by positively charging the conductor plate on the tunnel floor. These electrons move to the ground and are detectable as a current using a galvanometer integrated in the earth line. This idea is based on findings that the insect cuticle layer (the outer protective layer covering the body of many invertebrates) is highly conductive $e^{89-93}$ and that free electrons in this layer are affected by an electric field. ${ }^{67-70}$ The monitoring system can collect insect-specific signals continuously from discharge recorders set at multiple points in a warehouse. In addition to signal recognition, the correct identification of the pests is needed to determine the best way to eradicate them. As capturing pests is the most direct way to identify them, an electrostatic trap is attached to the discharge-detection box to catch insects passing over the discharge-detection plate. This trap is a simple modified version (two insulated conductor wires paired with an earthed metal plate) of the electrostatic insect-capturing instrument reported by Kakutani et al. ${ }^{67}$ The pest-monitoring apparatus consists of two electrostatic instruments: the insect-discharge detector and electrostatic insect trap. Both instruments are placed inside elongated rectangular boxes measuring $25 \mathrm{~cm}$ wide, $3 \mathrm{~cm}$ long, and $0.4 \mathrm{~cm}$ high, with an interior $0.2-\mathrm{cm}$-high tunnel allowing insect passage. The signal-generating part of the insect-discharge detector (black polypropylene) is an iron plate on the tunnel floor (Figure 3A). The plate is linked to a direct current positive voltage generator and positively charged with different voltages. Test insects (adults of cigarette beetle, red flour beetle, rice weevil) are released onto the charged plate, and the transfer of free electrons from the insect cuticle to ground is measured with a galvanometer integrated in the earth line of the voltage generator. The charge transfer is recorded as an electric current with a recorder built into the galvanometer. To construct the insect trap inside the box made of transparent acrylic, an earthed iron plate is installed on the tunnel floor, and two insulated conductors connect both ends of the tunnel ceiling (Figure 3B). The insulated conductor is prepared by passing an iron wire through a transparent insulator vinyl sleeve $\left(1 \mathrm{~mm}\right.$ thickness, $\left.1 \times 10^{9} \Omega \cdot \mathrm{cm}\right)$.

The two insulated conductors are connected to each other and a DC negative voltage generator and are negatively charged with 1.0 $10.0 \mathrm{kV}$. The negative surface charges of the insulated conductors polarize the earthed plate on the floor, creating a positive charge on the surface of the plat; the opposite charges act as dipoles that formed an electric field between the insulated conductors and earthed plate. Test insects are released onto the earthed plate to examine the ability of the insulated conductors to attract insects. All voltage generators used in this study are operated using $12-\mathrm{V}$ storage batteries. A monitoring is conducted using a pest-monitoring unit. The unit consists of two discharge detectors and the insect trap (Figure 3C). The boxes could all be connected to each other so that the passage floors are flat and continuous. Several pest-monitoring units are placed at several locations in the warehouse, and some opened bags of commercial dry-powdered diet for rats and rabbits are placed in the center of the warehouse to attract pests. All insect discharge data recorded in the galvanometers are transferred wirelessly to a radio receiver attached to a central computer for analyzing the movements of the pests (Figure 3D). The data are charted automatically for 7 days using data-analysis software. At the end of the monitoring period, the pests captured with the insulated conductors in the insect traps are counted and collected for identification. The bio-electrostatic nature of insects in an electric field can be used to construct an efficient system for specifically tracking the pests prowling in a warehouse stocking agricultural crops. The main goal of this study is to obtain continuous information on prowling pests automatically by collecting pest-specific signals from detectors set at multiple points in a warehouse. ${ }^{43}$ Biological discharge is the most reliable signal for this purpose, and our apparatus generates a single transient discharge signal from each pest entering a discharge detector. The electrostatic insect trap attached to the discharge detector enables us to identify the trapped pests. Using the pest-monitoring data, the nests and invasion routes of the pests are located, and chemical and physical measures are used to eradicate the pests nesting indoors and to prevent outdoor pests from entering the warehouse. 


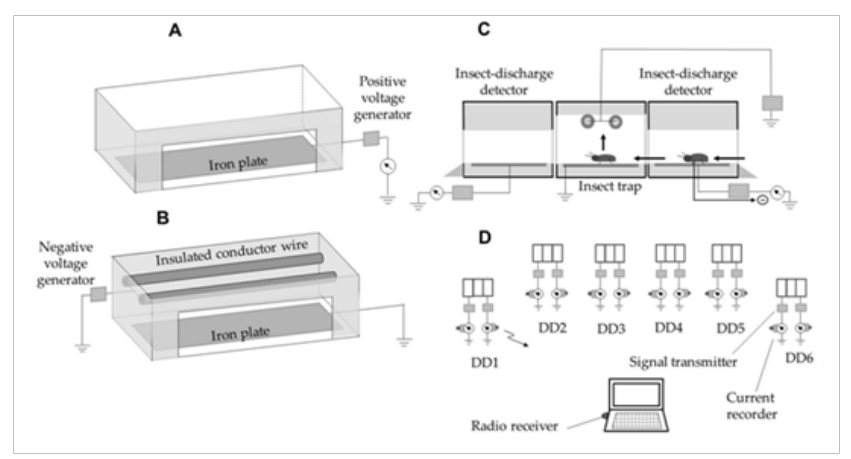

Figure 3 Diagram of the insect discharge detector $(A)$ and electrostatic insect trap (B) in an electrostatic pest monitoring system (C), and central control system for the insect discharge signals transmitted wirelessly from the multiple monitoring boxes set at different points (D). Bold arrows show the direction of insect movement or attraction, and the fine arrow indicates the direction of transfer of the insect charge.

\section{Construction of discharge-generating dipolar electric field screens}

\section{Ozone generative electric field screen}

In a non-uniform electric field, the charge on an insulator surface causes dipole in non-charged particles; the dipole develops a charge at one end and an equal amount and opposite charge at the other end of the particles. ${ }^{85}$ Powdery mildew conidia that came into this field also produce a dipole, and the opposite charges between the insulator and conidia create an electrostatic force. ${ }^{81}$ This electrostatic force is applicable to collecting conidia released from colonies of barley powdery mildew infecting host leaves ${ }^{82}$ and tomato powdery mildew. ${ }^{83}$ Based on these electrostatic characteristics, a cylindrical electrostatic spore precipitator (SM-screen) has been developed to control conidia of tomato powdery mildew $O$. neolycopersici. ${ }^{12}$ This spore precipitation cylinder can be improved to produce ozone, without spoiling its capability to attract air-borne conidia of powdery mildew. ${ }^{66}$ Our attention then turned to utilize ozone, a secondary product of the cylindrical spore precipitator, for the control of rhizosphere bacterial and fungal wilt pathogens (Ralstonia solanacearum and $F$. oxysporum f. sp. radicis-lycopersici) in hydroponic tomato. ${ }^{66} \mathrm{In}$ a nonuniform electric field, discharge occurs between oppositely charged conductors. ${ }^{78-80}$ Discharge causes not only the surrounding air to be ionized, but also generates ozone. ${ }^{94}$ The major aim of this work is to remodel an original spore precipitation cylinder into a new system that can produce ozone. For this, we utilize the center copper conductor wire of the cylinder as an anode and newly attach a cathodic copper ring to an edge of the cylinder.

The constructed cylinder is a new type of ozone generator that functions as an electrostatic spore precipitation cylinder. The operation of the new spore precipitation cylinders enables not only the trapping of conidia of tomato powdery mildew, but also allows for the control of rhizosphere pathogens entering the hydroponic culture system by the use of the ozone produced. The original cylinder of SM-screen contains a straight copper conductor wire covered with a transparent acrylic insulator cylinder and held with insulating silicon stoppers at the middle and at both ends of the cylinder. In the new cylinder, a silicon stopper at one end is set $10-\mathrm{cm}$ inside from the cylinder edge and a copper wire passed through it (Figure 4A). A copper ring, linked to an earthed line, is attached to a cylinder edge to cause a discharge between the wire end and the ring. Ozone gas is produced at the site of discharge (Site A in Figure 4A). Multiple cylinders are inserted into a polypropylene airflow pipe to collect the ozone produced. The inside air was transferred to an ozonometer or an air-mixing tank with an aspirator. A backflow preventer is set to the entrance of the airflow pipe to prevent the inside air from running back to the entrance when the aspirator stopped. The center conductor wires are linked in parallel to terminals of a connector line of an electrostatic voltage generator and are positively charged with different voltages. The charged wire creates a non-uniform electric field in a space between the wire and the inner surface of the cylinder to dielectrically polarize the cylinder (negative on the inner surface, positive on the outer surface) (Site B in Figure 4A), which produces a non-uniform electric field outside. This field is the region to create an attractive force between the cylinder and conidia that came into this field. ${ }^{12} \mathrm{~A}$ cylinder-edge ring (cathode) is changed 1 to $50 \mathrm{~mm}$ to produce different types of discharge in each voltage applied. Discharge is classified into four types according to a criterion described by ${ }^{95}$ corona, corona-streamer, streamer and arc (spark) discharges. A pale-blue light radiated by ionized air through the discharge is detected with a CCD camera using a previously described method.${ }^{96}$ For practical use, a cubic frame installed with the cylinders is attached to a hydroponic trough, and the ozone gas produced is transferred to a culture solution of the hydroponic trough.

The usual commercial apparatus for generating ozone are variations of a high-voltage electric discharge method. ${ }^{97}$ Our device is based on the same physical principle; a high voltage discharge site is prepared at the end of the spore precipitation cylinder. Discharge occurs in a non-uniform electric field between oppositely charged conductor electrodes, ${ }^{7-80}$ and during this stage the air in this field is ozonized. ${ }^{94}$ In the present device, the earthed ring and conductor wire tip work as cathodic and anodic electrodes, respectively, and generate an electric field in the space between the two electrodes. Our device generates distinct types of discharge (corona, corona-streamer, streamer and arc discharge), ${ }^{95}$ depending on the distances and potentials between the electrodes. Higher levels of ozone are generated through a streamer discharge. Ozone is an effective anti-microbial agent to kill viruses, bacteria and fungi in water ${ }^{98,99}$ and therefore has been used on its own to control rhizosphere pathogens in hydroponics ${ }^{100,101}$ or in combination with other chemical and physical techniques. ${ }^{102}$ An ozone sterilization technique has attracted great interest from growers, but commercial ozone generators are costly to be used as equipment for an agricultural use. Contrarily, our system is directly applicable to sterilization of the aqueous circumstances of hydroponic culture troughs furnished with the cylinders, since the spore precipitator cylinders can be constructed at a low cost. ${ }^{12}$ The use of this spore precipitator effectively prevents susceptible plants from becoming exposed to inoculum and is likely to lead to the lower use of chemicals. Such a result would find public acceptability for the use of this system as a non-chemical physical protector for crop plants.

\section{Electrostatic insect electrocution screen}

A novel electrostatic window screen was devised for installation in the windows of a piggery to prevent house mosquitoes (Culex pipiens) from transmitting Japanese encephalitis virus (JEV) to the surrounding human population. ${ }^{51}$ The structure of the electrostatic insect electrocution screen is shown in Figure 4B. Three identical stainless steel nets $(15 \mathrm{~mm}$ mesh) are arranged in parallel with $15-\mathrm{mm}$ spacing between nets to create opposite poles. The nets are mounted in a polycarbonate (insulator) frame that could be attached to a window. The central net $(\mathrm{CN})$ is connected to a direct-current voltage generator 
and negatively charged with different voltages; the external nets (ENs) are placed in front of and behind the $\mathrm{CN}$ and linked to an earth line. Polypropylene slips (insulators) $(15 \mathrm{~mm}$ thick) are placed between the nets as spacers to maintain the distance between electrodes (pole distance). The negative surface charge on the $\mathrm{CN}$ causes an electrostatic induction in the ENs, creating the opposite charge on the EN surface facing the $\mathrm{CN}$. An electric field forms between the oppositely charged $\mathrm{CN}$ and $\mathrm{EN}$. The transfer of free electrons from the $\mathrm{CN}$ to the $\mathrm{EN}$ is measured using two galvanometers integrated into the earth lines. The screen is negatively charged to produce a mechanical discharge (arc discharge) from the $\mathrm{CN}$ projection point, and a silent discharge, which is constantly generated from the EN-side surface of the $\mathrm{CN}$, is measured in the voltage range causing no mechanical discharge. The screen is negatively charged with different voltages to determine the range of voltages that cause electrocution in all insects introduced into the screen and the magnitude of the insect-mediated transient electric current (caused by arc discharge) at given voltages.

Our hypothesis is that a conductor material introduced into the space between the screen nets act as a temporary recipient of electrons from the $\mathrm{CN}$ and as a donor of electrons to the ENs due to the shorter pole distances. Insects are suitable bio-conductors for this purpose. Many previous studies ${ }^{89-93}$ have reported that the cuticle, an outer protective layer that covers the bodies of many invertebrates, is efficiently electrified due to its high conductivity. Adult house mosquitoes possess this cuticle structure. ${ }^{67}$ Once the insects are introduced into the electric field between the nets, they are subjected to an instantaneous and transient electric current by an arc discharge from the $\mathrm{CN}$ and violently ejected from the electric field. The insectmediated discharge is recorded as a transient electric current. This electrocution is selective due to the high conductivity of the insect cuticle, and the number of electrocuted insects increases as the applied voltage increased. Obviously, this device would also be effective for preventing insect pests from entering facilities (i.e., to keep animals free from various pests). Theoretically, the method is applicable to all insect pests that possess a conductive cuticle layer. In livestock pest control, ectoparasite control can be one of the most expensive and time-consuming activities, with a range of flies being common ectoparasites of animals in warmer climates. ${ }^{46}$ The proposed system is simple and easy to construct, and its scale could be increased through the use of larger nets corresponding to the size of the windows in specific livestock facilities.

Measurements with an ion detector showed that the electrostatic window screen ionized the air in the electric field with ozone generation. The ionized air in the electric field was extremely active and could destroy or inactivate various microorganisms, including viruses, and deodorize malodorous gases passing through the field. ${ }^{103}$ Various effluvia generated in the livestock facilities present serious environmental problems ${ }^{45}$; therefore, the ability of the current screen to remove odors may be a useful additional feature. In addition to its insect-electrocution ability, the device had several other advantageous characteristics that would justify its practical application in livestock facilities. The screen resulted in better air penetration for ventilation due to the use of spacious nets. The mesh size $(12 \mathrm{~mm})$ of the net was considerably larger than those ( $0.8-1.5 \mathrm{~mm}$ opening) of conventional woven insect nets. The installation of conventional woven insect nets to the openings of the facility used in this study caused a reduction in ventilation efficiency. ${ }^{45}$ The present screen functioned by preventing insect pests from getting into and out of the livestock facility, while maintaining strong air penetration. This is a vital outcome of the current study. To summarize, the novel electrostatic insect electrocution screen is a promising tool to create a pest-free space for raising animal livestock. The device could be constructed at low cost because of its very simple structure. The recent trend in urban livestock farming requires that virus-free animals be bred for supply in an environmentally controlled livestock facility, which could be facilitated by the use of the new device.

\section{Electrostatic smoke elimination screen}

Another type of the discharge-generating electric field screen was constructed to eliminate tobacco smoke. ${ }^{74}$ The screen consists of two non-insulated conductor poles; metallic pins, which are stuck with conductive double-side adhesive tape to a perforated polypropylene plate (insulator) with octagonal holes at constant intervals, and a stainless net arrayed in parallel with the plate (Figure 4C). The pin heads are connected to a direct-current voltage generator and negatively charged with different voltages, and the net linked to an earth line. In this electrostatic configuration, high voltages produced through a Cockcroft circuit $^{86}$ in the voltage generator are used to pick up electricity from a ground, to supply it to the spike tips of the plate, and their negative surface charge pushes free electrons of the net to the ground to create an opposite pole; an electric field forms between these opposite charges. Eventually, free electrons are transferred from the plate-side to the net-side ground during a corona discharge of the charged pin spikes. A corona discharge occurs when the strength (potential gradient) of the electric field around a pointed metal conductor is high enough to form a conductive region, but not to cause electrical breakdown or arcing to nearby objects ${ }^{104}$; the glow of the corona discharge is possibly photographed during a long exposure in a dark field. The present electrostatic device is configured so that two non-insulated conductor nets and spikes face each other to produce an electric field. In this electric field, a corona discharge constantly occurs from the spike tips of the plate to the net within the voltage range causing no arc discharge. The electric current produced by this discharge becomes greater in direct proportion to increases in the applied voltage in this voltage range.

An ionic wind is defined as the airflow induced by electrostatic forces linked to corona discharge arising at the tips of some sharp conductors (such as points or blades) subjected to high voltage relative to the ground. ${ }^{104}$ There is a clear correlation between the applied voltage and generation of ionic wind: the generation increases in direct proportion to the applied voltage. More importantly, larger voltages produce a stronger ionic wind involving more ions. In the present apparatus, the ionic wind is directed from the pointed tip of the plate toward the net. The ionic wind produced by the present apparatus is sufficiently strong $(1.5-2.0 \mathrm{~m} / \mathrm{sec})$ to bring outside air into the electric field of the screen. In an attempt to capture fine particles in smoke, our examination shows the positive correlation between negative ion generation and the trapping of fine smoke particles in the electric field of the present screen. These results strongly suggest the involvement of negative ions in the electrostatic attraction of smoke particles in the electric field of the screen. Thus, the results meet our expectation that negative ions can ionise smoke particles to generate an attractive force toward the opposite charge on the metal net. To summarise, our novel electrostatic apparatus is a promising device for creating a smoke-free space for non-smokers and preventing passive smoking. The device can be constructed cheaply because of its very simple structure and operated stably regardless of changes in environmental conditions such as temperature and humidity. 


\section{Related discharge-generating apparatuses}

\section{Electrostatic insect electrocution eliminator}

An electrostatic insect electrocution eliminator is the modified version of the electrostatic insect electrocution screen mentioned earlier. This eliminator was devised to selectively kill insect pests nesting in stored grains, seeds, and grain products. ${ }^{44}$ The structure of the apparatus is shown in Figure 4D. Two identical stainless steel nets are arranged in parallel with $10 \mathrm{~mm}$ spacing between nets to create opposite poles. One net $(\mathrm{CN})$ is connected to a direct-current voltage generator and negatively charged with different voltages, and another net (EN) is linked to an earth line. Polypropylene slips (insulators) (10 mm thick) are placed between the nets as spacers to maintain the distance between electrodes (pole distance). The negative surface charge on the $\mathrm{CN}$ causes an electrostatic induction in the EN, creating an opposite surface charge on the $\mathrm{CN}$-side surface of the EN. An electric field forms between these opposite charges on $\mathrm{CN}$ and $\mathrm{EN}$. The transfer of free electrons from the $\mathrm{CN}$ side to the EN-side ground is measured using two galvanometers integrated into the grounded lines. The outside of the apparatus is covered with a polypropylene insulator to prevent rice grains from leaking through the mesh or from the side edges. Test insects, adult rice weevils are introduced into the electric field between the nets. Once the insects are introduced, they are subjected to instantaneous and transient electric current by an arc discharge from the $\mathrm{CN}$, and violently ejected from the electric field. These insects are electrocuted, and the number of dismembered insects increase as the applied voltage increased. This exterminatory effect is similarly detected when a mixture of rice grains and insect pests is introduced into the electric field.

Pests are selectively electrocuted within the apparatus due to the high conductivity of the insect cuticle and the insulative properties of rice grains. However, the magnitude of the electric current is conspicuously different among the sizes of the apparatuses used, even when the same voltage is applied. Obviously, different amounts of electricity (free electrons) accumulate on the $\mathrm{CN}$ and are released toward the insect. The magnitude of the increase in current corresponds to increases in $\mathrm{CN}$ area (i.e., increases in the electrical capacitance of the $\mathrm{CN}$ ). The proposed system is simple and easy to construct, and its scale can be enlarged through the use of larger nets, corresponding to the volume of rice grains. Thus, the present study provides an experimental basis for the practical application of an electrostaticsbased pest control method. Based on these results, we are able to apply our method to the eradication of rice weevils nesting in bagged rice $(20 \mathrm{~kg})$ for commercial use, which had been rejected on a regular inspection for contamination by insect pests. In this approach, we use the large apparatus (charged with $9 \mathrm{kV}$ ) to enhance our ability to treat rice grains. All rice grains in each bag are treated within a short time (10-11 min), and all insects are crushed without leaving any trace. Judging from these results, we propose the most effective application of the present system; this method could be integrated into the line between the rice polishing and packaging processes for the preparation of pest-free bagged rice. Theoretically, the present method is applicable to all insect pests that possess a conductive cuticle layer; during the preliminary assay, we confirm successful application to the control of stored product pests, such as the cigarette beetle, red flour beetle and Azuki bean weevil.

\section{Corona discharge exposing probe}

A corona discharge exposing probe is an apparatus to eradicate powdery mildew colonies on leaves by a corona discharge (plasma stream $)^{105}$ produced between the pointed tip of electrified conductor and the earthed plant leaf (Figure. 4E). ${ }^{13}$ In this apparatus, a copper wire with a pointed tip is used as a cathodic conductor probe. Except for the pointed tip, the wire is insulated with a vinyl sleeve and linked to a voltage generator. The conductor probe is coaxially held in an acrylic cylinder with an insulating silicon stopper and negatively charged with a potential of 5 to $30 \mathrm{kV}$. An electric field between the leaf and the charged probe tip is formed by touching an earthed electric wire to the branch of the test leaf. The pale-blue light radiated by ionized air through corona discharges is detected in the dark. A corona, characterized by a blue glow, forms at the needle tip, as the probe is brought closer to a leaf surface. The distance at which this occurs increased from $16-50 \mathrm{~mm}$ as the voltage is increased from $5-30 \mathrm{kV}$. If the probe is brought too close to the leaf surface, an arc discharge occurs which caused injury to the leaf. Powdery mildew colonies are destroyed by 2 second exposures at probe distances where corona discharge is initiated; a probe distance of $25 \mathrm{~mm}$ and $30 \mathrm{kV}$ for a 2-second burst is the suitable condition for controlling powdery mildew in a greenhouse environment. The corona discharge generator is portable and easy to operate, and the exposure treatment could be integrated as a part of routine crop care; the corona discharge exposure can be applied as soon as powdery mildew colonies are visible. In these colonies, conidia on conidiophores are immature, so killing them at this stage effectively suppresses the subsequent production and release of mature conidia. ${ }^{106,107}$ Thus, the corona discharge generator is a promising alternative disease control tool for complete eradication of powdery mildew without fungicidal treatment.
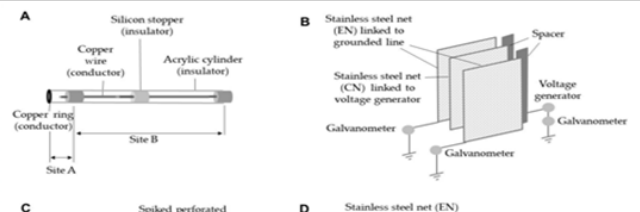

c
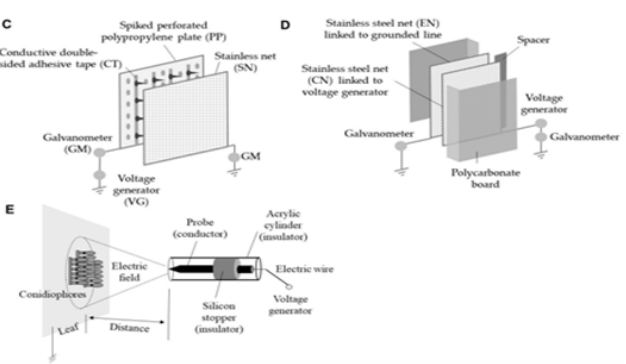

Figure 4 Structure of ozone generative cylinder of modified SM-screen (A), electrostatic insect electrocution screen (B), electrostatic smoke eliminator screen (C), electrostatic smoke elimination screen (D), electrostatic insect electrocution eliminator (E), and corona discharge exposing probe (D).

\section{Conclusion}

Applied electrostatic engineering has served as the academic and technical basis for our various methods that successfully manage airborne biotic and abiotic nuisances in various environments. The ways in which electrostatic principles have been applied are diverse and include the following: capturing spores and insects by exploiting the attractive force generated in a static electric field (without electric discharge); repelling insects according to their aversion to the electric field. A discharge generation is an alternative way to produce an attractive force by ionizing fine particles with negative ions produced in the discharge generating electric field. In addition, other electric discharge techniques were useful to disinfect bacterial and fungal pathogens using ozone produced through streamer discharge, instantaneously dislodge fungal pathogens from 
plants through exposure to a plasma stream produced via a corona discharge in the electric field, and selectively electrocute insect pests nesting in cereal grains and house mosquitoes transmitting pathogens to the surrounding human population. Based on these successful applications, we have proposed various electrostatic apparatuses to manage numerous environmental nuisances. Most serious nuisances are aerial spores of plant pathogens and small flying insect pests in agricultural and horticultural environments. In fact, the crop plants suffer from pathogen infection and/or insect attack during their preharvest and post-harvest stages. Chemical, biological and physical methods have been developed to prevent these attacks. However, excessive application of agrochemicals such as fungicides and insecticides often causes chemical resistance or less sensitive mutants of the pathogens and pest insects and/or environmental pollution, and biological controls using antagonistic microbes or natural enemies are not always effective and stable in their suppressive effects. Insect-excluding woven screens with a fine mesh size have been a conventional physical method to minimise insect entry to glasshouses, but the disadvantage of screening is a reduction in ventilation that can cause overheating and increase relative humidity. In the interest of protecting crops during production and storage, we have developed electrostatic methods to disinfect bacterial and fungal plant pathogens or to prevent airborne pathogens and flying insect pests from entering greenhouses, with the aim of reducing the use of fungicides and insecticides. An electric field screen has been practically used as an environmentally friendly tool to exclude pathogens and pests from spaces of plant cultivation and storage with better air penetration. This technique has been developed to trap other aerial biotic and abiotic nuisances in various human-life environments.

\section{Acknowledgements}

None.

\section{Conflict of interest}

The author declares there is no conflict of interest.

\section{References}

1. Toyoda H, Matsuda Y, Nonomura T. Comprehensive approaches to crop protection. RAEFSS publishing Nara Jpn. 2015;1-316.

2. Toyoda H, Matsuda Y, Nonomura T. Molecular approaches to chemical and biological control of bacterial wilt pathogen of tomato. In Plant diseases and their control. China Agricultural Scientech Press, Beijing, China, 2000; pp.79-82.

3. Toyoda H, Matsuda Y. Electric field screen; principles and applications. Nobunkyo Production, Tokyo, Jpn, 2015; pp.1-238.

4. Kiss L, Cook RTA, Saenz GS. Identification of two powdery mildew fungi, Oidium neolycopersici sp. nov and O. lycopersici, infecting tomato in different parts of the world. Mycol Res. 2001;105:684-697.

5. Kashimoto K, Sameshima T, Matsuda Y, et al. Infectivity of a Japanese isolate of Oidium neolycopersici KTP-01 to a European tomato cultivar resistant to O. lycopersici. J Gen Plant Pathol. 2003;69(6):406-408.

6. Lindhout P, Pet G, van der Beek H. Screening wild Lycopersicon species for resistance to powdery mildew (Oidium lycopersicum). Euphytica. 1994;72(1-2):43-49.

7. Mieslerová B, Lebeda A, Chetelat RT. Variation in response of wild Lycopersicon and Solanum spp. against tomato powdery mildew (Oidium lycopersici). J Phytopathol. 2000;148(5):303-311.

8. van der Beek JG, Pet G, Lindhout P. Resistance to powdery mildew
(Oidium lycopersicum) in Lycopersicon hirsutum is controlled by an incompletely-dominant gene $\mathrm{Ol-1}$ on chromosome 6. Theor Appl Genet. 1994;89(4):467-473.

9. Brown JKM. Comparative genetics of avirulence and fungicide resistance in the powdery mildew fungi. In The Powdery Mildews, Bélanger RR. et al., editors. American Phytopathological Society Press: St. Paul, USA, 2002; pp.56-65.

10. Lebeda A, Mieslerová B. Variability in pathogenicity of Oidium neolycopersici on Lycopersicon species. J Plant Dis Prot. 2002;109(2):129-141.

11. Matsuda Y, Mori Y, Nishida M, et al. Screening of wild Lycopersicon species for resistance to Japanese isolate of tomato powdery mildew Oidium neolycopersici. Breeding Sci. 2005;55:355-360.

12. Matsuda Y, Ikeda $\mathrm{H}$, Moriura N, et al. A new spore precipitator with polarized dielectric insulators for physical control of tomato powdery mildew. Phytopathology. 2006;96:967-974.

13. Nonomura T, Matsuda Y, Kakutani K, et al. Physical control of powdery mildew (Oidium neolycopersici) on tomato leaves by exposure to corona discharge. Can J Plant Pathol. 2008;30(4):517-524.

14. Kakutani K, Matsuda Y, Nonomura T, et al. Practical application of an electric field screen to an exclusion of flying insect pests and airborne conidia from greenhouses with a good air penetration. J Agric Sci. 2012;4(5):51-60.

15. Takikawa Y, Matsuda Y, Nonomura T. An electrostatic nursery shelter for safely raising pest-free and pathogen-free tomato seedlings in a window-open greenhouse. J Agric Sci. 2015;8:1-25.

16. Sequeira S, Cabrita EJ, Macedo MF. Antifungals on paper conservation: An overview. Int Biodeterior Biodegradation. 2012;74:67-86.

17. Takikawa Y, Matsuda Y, Nonomura T, et al. Electrostatic guarding of bookshelves from mould-free preservation of valuable library books. Aerobiologia. 2014;30(4):435-444.

18. da Silvia M, Morgaes A.L, Nishikawa MM, et al. Inactivation of fungi from deteriorated paper materials by radiation. Int Biodeterior Biodegradation. 2006;57:163-167.

19. Kanetis L, Forster H, Adaskaveg JE. Determination of natural resistance frequencies in Penecillium digitatum using a new air-sampling method and characterization of fluodioxonil- and pyrimethanil-resistant isolates. Phytopathology. 2010;100(8):738-746.

20. Foote BA. Biology of shore flies. Ann Rev Entomol. 1995;40:417-442.

21. Helyer N, Brown K, Cattlin ND. Pest profiles. In A colour handbook of biological control in plant protection. Northcott J editor. Manson Publishing, London, UK, 2004; pp.21-41.

22. Hill DS. Pests of stored products and their control. Belhaven Press, London, UK, 1990; pp.1-274.

23. Foster WA, Walker ED. Mosquitoes (Culicidae). In Medical and veterinary entomology. Elsevier Science, New York, USA, 2002; pp.203-262.

24. Tanaka N, Matsuda Y, Kato E. An electric dipolar screen with oppositely polarized insulators for excluding whiteflies from greenhouses. Crop Prot. 2008; 27:215-221.

25. Gillespie DR, Menzies JG. Fungus gnats vector Fusarium oxysporum f. sp. radicis-lycopersici. Ann Appl Biol. 1993;123(3):539-544.

26. El-Hamalawi ZA. Attraction, acquisition, retention and spatiotemporal distribution of soilborne plant pathogenic fungi by shore flies. Аnпи Appl Biol. 2007;152(2):169-177.

27. Perring TM. The Bemisia tabaci species complex. Crop Prot. 


\section{$2001 ; 20: 725-737$}

28. Cohen S, Berlinger J. Transmission and cultural control of white-fly borne viruses. Agric Ecosyst Environ. 1986;17:89-97.

29. Oliveira MRV, Henneberry TJ, Anderson P. History, current status, and collaborative research projects for Bemisia tabaci. Crop Prot. 2001;20:709-723

30. Sharaf N. Chemical control of Bemisia tabaci. Agric Ecosyst Environ 1986;17(1-2):111-127.

31. Prabhaker N, Coudriet DL, Meyerdirk DE. Insecticide resistance in the sweetpotato whitefly, Bemisia tabaci (Homoptera: Aleyrodiae). J Econ Entomol. 1985;78:748-752.

32. Palumbo JC, Horowitz AR, Prabhaker N. Insecticidal control and resistance management for Bemisia tabaci. Crop Prot. 2001;20(9):739765 .

33. Horowitz AR, Kontsedalov S, Ishaaya, I. Dynamics of resistance to the neonicotinoids acetamiprid and thiamethoxam in Bemisia tabacci (Homoptera: Aleyrodidae). J Econ Entomol. 2004;97(6):2051-2056.

34. Nauen R, Denholm I. Resistance of insect pests to neonicotinoid insecticides: Current status and future respects. Adv Insects Biochem Physiol. 2005;58(4):200-215.

35. Weintrub PG, Berlinger MJ. Physical control in greenhouses and field crops. In Insect Pest Management. Horowitz AR, Ishaaya I, Eds, Springer-Verlag, Amsterdam, Netherland, 2004; pp.301-318.

36. Ueda S, Brow JK. First report of the Q biotype of Bemisia tabaci in Japan by mitochondrial cytochrome oxidase I sequence analysis. Phytoparasitica. 2006;34(4):405-411.

37. Nonomura T, Matsuda Y, Kakutani K, et al. An electric field strongly deters whiteflies from entering window-open greenhouses in an electrostatic insect exclusion strategy. Europ J Plant Pathol. 2012;134:661-670.

38. Kakutani K, Matsuda Y, Takikawa Y. Successful single-truss cropping cultivation of healthy tomato seedlings raised in an electrostatically guarded nursery cabinet with non-chemical control of whiteflies. Glob J Pest Dis Crop Prot. 2017;5:269-275.

39. Cayol JP, Causse R, Louis C, et al. Medfly Ceratitis capitata Wiedemann (Dipt., Trypetidae) as a rot vector in laboratory conditions. J Appl Entomol. 1994;117:338-343.

40. Sela S, Nestel D, Pinto R, et al. Mediterranean fruit fly as a potentia vector of bacterial pathogens. Appl Environ Microbiol. 2005;71:40524056.

41. Janisiewicz WJ, Conway WS, Brown MW, et al. Fate of Escherichia coli $\mathrm{O} 157: \mathrm{H} 7$ on fresh-cut apple tissue and its potential for transmission by fruit flies. Appl. Environ. Microbiol. 1999;65(1):1-5.

42. Matsuda Y, Nonomura T, Kakutani K, et al. A newly devised electric field screen for avoidance and capture of cigarette beetles and vinegar flies. Crop Prot. 2011;30(2):155-162.

43. Takikawa Y, Matsuda Y, Nonomura T. Electrostatic trap with an insect discharge recorder for multiple real-time monitoring of pests prowling in a warehouse. Int J Adv Agric Res. 2015;3:55-63.

44. Matsuda Y, Takikawa Y, Nonomura T, et al. Selective electrostatic eradication of Sitopholus oryzae nesting in stored rice. J Food Technol Preserv. 2018;2(1):15-20.

45. Gates RS, Casey KD, Xin H, et al. Fan Assessment numeration system (FANS) design and calibration specifications. Transactions of the ASAE. 2004;47:1709-1715.

46. Hurk AF, Ritchie SA, Johansen CA, et al. Domestic Pigs and
Japanese Encephalitis Virus Infection, Australia. Emeg. Infect. Dis. 2008;14(11):1736-1738.

47. Uchil PD, Satchidanandam V. Phylogenetic analysis of Japanese encephalitis virus: envelope gene based analysis reveals a fifth genotype geographic clustering, and multiple introductions of the virus into the Indian subcontinent. Am J Trop Med Hyg. 2001;65(3):242-251.

48. Rosen L. The natural history of Japanese Encephalitis Virus. Annu Rev Microbiol. 1986;40:395-414.

49. Tiwari S, Singh RK, Tiwari R, et al. Japanese encephalitis: a review of the Indian perspective. Braz J Infect Dis. 2012;16(6):564-573.

50. Lindahl JF, Chirico J, Boqvist S, et al. Occurrence of Japanese encephalitis virus mosquito vectors in relation to urban pig holdings. Am J Trop Med Hyg. 2012;87(6):1076-1082.

51. Kakutani K, Matsuda Y, Takikawa Y. Electrocution of mosquitoes by a novel electrostatic window screen to minimize mosquito transmission of Japanese encephalitis viruses. Int J Sci Res. 2018;7:47-50.

52. Bobanga T, Ayieko W, Zanga M, et al. Field efficacy and acceptability of PermaNet 3. $0^{\circledR}$ and OlysetNet ${ }^{\circledR}$ in Kinshasa, Democratic Republic of the Congo. J Vector Borne Dis. 2013;50(3):206-214.

53. N'Guessan R, Corbel V, Akogbéto M, et al. Reduced efficacy of insecticide-treated nets and indoor residual spraying for malaria control in pyrethroid resistance area Benin. Infect Dis. 2007;13(2):199-206.

54. Sharma SK, Tyagi PK, Upadhyay AK, et al. Efficacy of permethrin treated long-lasting insecticidal nests on malaria transmission and observations on the perceived side effects, collateral benefits and human safety in a hyperendemic tribal area of Orissa, India. Acta Trop. 2009;112(2):181-187.

55. Matsuda Y, Kakutani K, Nonomura T. Safe housing ensured by an electric field screen that excludes insect-net permeating haematophagous mosquitoes carrying human pathogens. J Phys (Conference series). 2015;646:0120021-0120024.

56. Kaneko $\mathrm{Y}$, Motohashi $\mathrm{Y}$, Nakamura $\mathrm{H}$, et al. Increasing prevalence of Japanese cedar pollinosis: A meta-regression analysis. Int. Arch. Allergy Immunol. 2005;136(4):365-371

57. Matsuda Y, Kakutani K, Nonomura T, et al. An oppositely charged insect exclusion screen with gap-free multiple electric fields. J. Appl Phys. 2012;112(11):116103(-1) -116103(-3).

58. Nonomura T, Matsuda Y, Kakutani K, et al. Prevention of whitefly entry from a greenhouse entrance by furnishing an airflow-oriented pre-entrance room guarded with electric field screens. J Agric Sci. 2014;6(12):172-184

59. Takikawa Y, Matsuda Y, Nonomura T, et al. An electrostatic-barrierforming window that captures airborne pollen grains to prevent pollinosis. Int J Environ Res Pub Health. 2017;14(1):1-5.

60. Schlesinger RB. The Health Impact of Common Inorganic Components of Fine Particulate Matter (PM2.5) in Ambient Air: A Critical Review. Inhal Toxicol. 2007;19(10):811-832.

61. Takikawa Y, Matsuda Y, Nonomura T. Electrostatic elimination of fine smoke particles by a newly devised air purification screen. Int J Sci Res Environ Sci. 2017;51:17-21.

62. Cao S, Yang C, Gan Y, et al. The health effects of passive smoking: An overview of systematic reviews based on observational epidemiological evidence. PLOS ONE. 2015;10(10):e0139907.

63. Dinas PC, Koutedakis Y, Flouris AD. Effects of active and passive tobacco cigarette smoking on heart rate variability. Int $J$ Cardiol. 2013;163(2):109-115.

64. Cui H, Gong TT, Liu CX, et al. Associations between passive maternal 
smoking during pregnancy and preterm birth: Evidence from a metaanalysis of observational studies. PLOS ONE. 2016;11(1):e0147848.

65. Saulyte J, Regueira C, Montes-Martínez A, et al. Active or passive exposure to tobacco smoking and allergic rhinitis, allergic dermatitis, and food allergy in adults and children: A systematic review and metaanalysis. PLoS Med. 2014;11(3):e1001939.

66. Shimizu K, Matsuda Y, Nonomura T, et al. Dual protection of hydroponic tomatoes from rhizosphere pathogens Ralstonia solanacearum and Fusarium oxysporum f. sp. radicis-lycopersici and airborne conidia of Oidium neolycopersici with an ozone-generative electrostatic spore precipitator. Plant Pathol. 2007;56(6):987-997.

67. Kakutani K, Matsuda Y, Haneda K, et al. An electric field screen prevents captured insects from escaping by depriving bioelectricity generated through insect movements. J Electrostat. 2012;70(2):207211.

68. Kakutani K, Matsuda Y, Haneda K, et al. Insects are electrified in an electric field by deprivation of their negative charge. Ann. Appl. Biol. 2012;160(3):250-259

69. Matsuda Y, Nonomura T, Kakutani K, et al. Avoidance of an electric field by insects: Fundamental biological phenomenon for an electrostatic pest-exclusion strategy. $J$ Phys (Conference Series). 2015;646:0120031-0120034.

70. Nonomura T, Matsuda Y, Kakutani K, et al. Electrostatic measurement of dischargeable electricity and bioelectric potentials produced by muscular movements in flies. J. Electrostat. 2014;72(1):1-5.

71. Takikawa Y, Matsuda Y, Kakutani K, et al. Electrostatic insect sweeper for eliminating whiteflies colonizing host plants; a complementary pest control device in an electric field screen-guarded greenhouse. Insects. 2015;6(2):442-454.

72. Takikawa Y, Matsuda Y, Nonomura T, et al. Elimination of whiteflies colonizing greenhouse tomato plants using an electrostatic flying insect catcher. Int J Cur Adv Res. 2017;6(2):5517-5521.

73. Takikawa Y, Matsuda Y, Nonomura T. Exclusion of whiteflies from a plastic hoop greenhouse by a bamboo blind-type electric field screen J Agric Sci. 2018

74. Matsuda Y, Takikawa Y, Nonomura T. A simple electrostatic device for eliminating tobacco sidestream to prevent passive smoking. Instruments. 2018;2:3:1-10.

75. Matsuda Y, Sameshima T, Moriura N, et al. Identification of individual powdery mildew fungi infecting leaves and direct detection of gene expression by single conidium PCR. Phytopathology. 2005;95(10):1137-1143.

76. Moore AD. Frictional electricity: In Electrostatics, exploring, controlling, and using static electricity 2nd edition. Laplacian Press, California, USA, 1997; pp.22-27.

77. Jones E. Childers R. Electric charge and electric field. In Physics. McGraw-Hill, Boston, USA, 2002; pp. 495-525.

78. Griffith WT. Electrostatic phenomena. In The Physics of Everyday Phenomena, A Conceptual Introduction to Physics. Bruflodt D, et al., Editors. McGraw-Hill, New York, USA, 2004; pp. 232-252.

79. Giancoli DC. Electric charge and electric field. In: Physics, Principles with Applications. Challice J. editor. Pearson Education International, London, UK, 2005; pp. 39-469.

80. Halliday D, Resnick R, Walker J. Electric discharge and electric fields. In Fundamentals of Physics. Johnson S, et al., editors. John Wiley \& Sons, New York, USA, 2005; pp. 561-604.

81. Moriura N, Matsuda Y, Oichi W, et al. Consecutive monitoring of lifelong production of conidia by individual conidiophores of Blumeria graminis f. sp. hordei on barley leaves by digital microscopic techniques with electrostatic micromanipulation. Mycol Res. 2006;110:18-27.

82. Moriura N, Matsuda Y, Oichi W, et al. An apparatus for collecting total conidia of Blumeria graminis f. sp. hordei from leaf colonies using electrostatic attraction. Plant Pathol. 2006;55(11):367-374.

83. Nonomura T, Matsuda Y, Xu L. Collection of highly germinative pseudochain conidia of Oidium neolycopersici from conidiophores by electrostatic attraction. Mycol Res. 2009;113:364-372.

84. Kaiser KL. Air breakdown. In Electrostatic discharge. Taylor \& Francis, New York, USA, 2006; pp.1-102.

85. Cross JA. Dielectrophoresis. In Electrostatics: Principles, Problems and Applications. De Barr AE. Ed, Adam Hilger, Bristol, USA, 1987; pp.269-276.

86. Wegner HE. Electrical charging generators. In McGraw-Hill Encyclopedia of Science and Technology-9th Edition. Geller E. et al editors. The Lakeside Press, New York, USA, 2002; pp.42-43.

87. Burkholder WE. Stored product insect behavior and pheromone studies: keys to successful monitoring and trapping. Proc. of the 3rd International Conference of Stored Product Entomology. Univ. of Kansas, Manhattan, USA, 1984; pp.20-33.

88. Klotz JH, Reid BL. The use of spatial cues for structural guidline orientation in Tapinoma sessile and Camponotus pennsylvanicus (Hymenoptera: Formicidae). J Insect Behav. 1992;5(1):71-82.

89. Ishay JS, Shimony TB, Shalom AB, et al. Photovoltaic effects in the oriental hornet, Vespa orientalis. J Insect Physiol. 1992;38(1):37-48.

90. McGonigle DG, Jackson CW, Davidson JL. Triboelectrification of houseflies (Musca domestica L.) walking on synthetic dielectric surfaces. J Electrostat. 2002;54:167-177.

91. McGonigle DG, Jackson CW. Effect of surface material on electrostatic charging of houseflies (Musca domestica L). Pest Manag Sci. 2002;58(4):374-380

92. Honna T, Akiyama Y, Morishima K. Demonstration of insect-based power generation using a piezoelectric fiber. Comp. Biochemi. Physiol. Part B: Biochem Mol Biol. 2008; 151:460.

93. Moussian B. Recent advances in understanding mechanisms of insect cuticle differentiation. Insect Biochem Mol Biol. 2010;40(5):363-375.

94. Chen J, Davidson JH. Ozone production in the positive DC corona discharge: Model and comparison to experiments. Plasma Chem Plasma P. 2002;22(2):495-522.

95. Goelian, N, Lalande P, Bondiou-Clergerie A. A simplified model for the simulation of positive-spark development in long air gaps. $J$ Phys D: Appl Phys. 1997;30:2441-2452.

96. van Veldhuizen EM, Rutgers WR. Pulsed positive corona streamer propagation and branching. J Phys D: Appl Phys. 2002;35:2169-2179.

97. Takayama M, Ebihara K, Stryczewska H, et al. Ozone generation by dielectric barrier discharge for soil sterilization. Thin Solid Films. 2006;506-507:396-399.

98. Burleson GR, Murray TM, Pollard, M. Inactivation of viruses and bacteria by ozone, with and without sonication. App. Microbiol. 1975;29(3):340-344.

99. Farooq S, Chian ESK, Engelbrecht RS. Basic concepts in disinfection with ozone. J Water Pollu Cont Fed. 1977;49:818-1831.

100. Vanachter A, Thys L, Van Wambeke E. Possible use of ozone for disinfestation of plant nutrient solutions. Acta Hort. 1988;221:295-302. 
101. Runia WTH. A review of possibilities for disinfection of recirculation water from soilless cultures. Acta Hort. 1995;382:221-229.

102. van Os EA. Design of sustainable hydroponic systems in relation to environment-friendly disinfection methods. Acta Hort. 2001; 548:197205.

103. Nishizawa K, Nojima H. Air purification effect of positively and negatively charged ions generated by discharge plasma at atmospheric pressure. Jpn. J. Appl. Phys. 2001; 40:835-837.

104. Jonassen N. Electrostatic effects and Abatement of static electricity. In Electrostatics. Kluwer Academic Publishers, Massachusetts, USA 2002; pp.75-120.
105. Herrmann HW, Henins I, Park J, et al. Decontamination of chemical and biological warfare (CBW) agents using an atmospheric pressure plasma jet (APPJ). Phys Plasmas. 1999;6(5):2284-2289.

106. Oichi W, Matsuda Y, Sameshima T, et al. Consecutive monitoring for conidiogenesis by Oidium neolycopersici on tomato leaves with a high-fidelity digital microscope. J Gen Plant Pathol. 2004;70(6)329-332.

107. Oichi W, Matsuda Y, Nonomura T, et al. Formation of conidial pseudochains by tomato powdery mildew Oidium neolycopersici. Plant Dis. 2006;90:915-919. 